\title{
Optical extinction and scattering cross sections of plasmonic nanoparticle dimers in aqueous suspension
}

Matthieu Loumaigne[1], Clyde Midelet[1], Tristan Doussineau[2], Philippe Dugourd[2],

Rodolphe Antoine[2], Meriem Stamboul[3], Anne Débarre[3][4] and

Martinus H. V. Werts*[1]

[1] École normale supérieure de Rennes, CNRS, SATIE (UMR 8029), Campus de Ker Lann, F35170 Bruz, France

[2] Institut Lumière Matière, UMR CNRS 5306 and Université Claude Bernard Lyon 1, Université de Lyon, 69622 Villeurbanne cedex, France

[3] Laboratoire Aimé Cotton, CNRS, Univ. Paris-Sud, ENS Cachan, Université Paris-Saclay, 91405 Orsay Cedex, France

[4] École normale supérieure de Cachan, CNRS, PPSM (UMR 8531), F-94235 Cachan, France.

*) Corresponding author; e-mail: martinus.werts@ens-rennes.fr

\section{ACCEPTED MANUSCRIPT - AUTHOR'S VERSION}

Final published version can be found at https://doi.org/10.1039/C6NR00918B

Citation: $\quad$ M. Loumaigne, C. Midelet, T. Doussineau, P. Dugourd, R. Antoine, M. Stamboul, A. Débarre, M. H. V. Werts, Nanoscale 2016, 8, 6555-6570

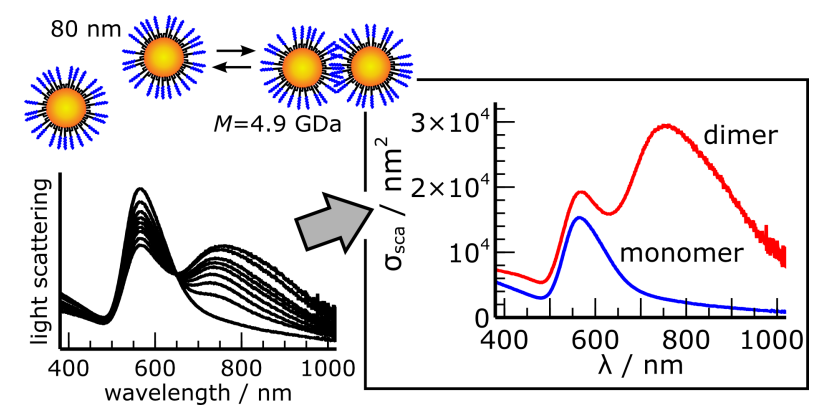

Absolute extinction and scattering cross sections of gold nanoparticle dimers were determined experimentally using a chemometric approach. 


\section{Abstract}

Absolute extinction and scattering cross sections for gold nanoparticle dimers were determined experimentally using a chemometric approach involving singular-value decomposition of the extinction and scattering spectra of slowly aggregating gold nanospheres in aqueous suspension. Quantitative spectroscopic data on plasmonic nanoparticle assemblies in liquid suspension are rare, in particular for particles larger than 40 $\mathrm{nm}$, and in this work we demonstrate how such data can be obtained directly from the aggregating suspension. Our method can analyse, non invasively, the evolution of several sub-populations of nanoparticle assemblies. It may be applied to other self-assembling nanoparticle systems with an evolving optical response. The colloidal systems studied here are based on 20, 50 and $80 \mathrm{~nm}$ gold nanospheres in aqueous solutions containing sodium lipoate. In these systems, the reversible dimerisation process can be controlled using $\mathrm{pH}$ and ionic strength, and this control is rationalised in terms of DLVO theory. The dimers were identified in suspension by their translational and rotational diffusion through scattering correlation spectroscopy. Moreover, their gigadalton molecular weight was measured using electrospray charge-detection mass spectrometry, demonstrating that mass spectrometry can be used to study nanoparticles assemblies of very high molecular mass. The extinction and scattering cross sections calculated in the discrete-dipole approximation (DDA) agree very well with those obtained experimentally using our approach.

\section{Introduction}

The aggregate of two spherical plasmonic nanoparticles (henceforth referred to as nanosphere (NS) dimer, or simply dimer) is a fundamental building block in the construction of plasmonic assemblies. ${ }^{1-6}$ Additionally, the formation of dimers represents the first step in the aggregation of colloidal particles in suspension..$^{7-10}$ When mediated by suitable (bio)molecules or (bio)chemical stimuli, such aggregation is at the basis of a large variety of biological and chemical sensing schemes using suspensions of small latex particles, ${ }^{11,12}$ or gold or silver nanoparticles. ${ }^{13-20}$ In the case of plasmonic nanoparticles, the interaction of the optical resonances of the constituent particles in the dimer leads to radical changes in the 
optical absorption and scattering spectra ${ }^{15,21-23}$ as a result of the formation of a longitudinal plasmon resonance at longer wavelengths. This is illustrated by the well-known colour change from red to blue upon salt-induced aggregation of citrate-stabilised gold NS in suspension..$^{24,25}$

In the past, particle dimers have been synthesised and separated from the monomers and trimers present in the mixtures. ${ }^{15,26-29}$ These dimeric objects can be redispersed in aqueous suspension for further spectroscopic study. However, quantitative measurements of optical cross sections on such samples have not been obtained due to the lack of precise knowledge of the concentrations of the objects. Absolute optical cross sections of individual objects can be measured by nanospectroscopic techniques applied to samples deposited on a suitable substrate,$^{30}$ but this does not give access to the optical response of the objects in their native homogeneous and isotropic liquid environment, and requires measurement of many individual objects for statistical comparison.

Here, we present a physicochemical and optical spectroscopic study of dimers in aqueous systems containing colloidal plasmonic nanospheres of various diameters and lipoic acid (LA, also called thioctic acid) at varying $\mathrm{pH}$. The functionalization of gold NS with LA ${ }^{31}$ yields particles that we demonstrated ${ }^{22}$ to undergo reversible aggregation in acidic environment (typically, at $\mathrm{pH}$ values below the $\mathrm{pK}_{\mathrm{a}}$ value of lipoic acid). The colloidal chemistry of this system is studied in more detail by varying $\mathrm{pH}$, ionic strength, and also ligand (LA) concentration. The aqueous gold nanosphere-lipoic acid system turns out to be a highly versatile plasmonic colloidal system demonstrating controllable and reversible aggregration of nanospheres over a wide range of NS diameters $(15 \ldots 80 \mathrm{~nm})$. Assembly of gold particles with diameters larger than $40 \mathrm{~nm}$ is relevant for biosensing using plasmonic light scattering, and is at present not readily achieved by other nano-assembly strategies such as DNA-based assembly. ${ }^{15,26,29}$ The aggregation is considered in the light of the DLVO theory, and we suggest a modified potential energy curve to account for the lipoic ligand shell that confers reversibility to the aggregation of the gold nanoparticles.

By applying chemometric multicomponent analysis to the optical spectra of aggregating NS suspensions, we obtain absolute extinction and scattering cross section spectra of all species present as well as information on the relative populations of monomers and dimers. Such spectroscopic data is valuable for confronting theoretical models of plasmonic interactions and for the evaluation of the analytical performance of aggregation-based plasmonic 
biosensing schemes. At present, quantitative data on optical cross sections of plasmonic nanoparticle assemblies have only been obtained for individual dimers deposited on dry substrates, ${ }^{32}$ and not for such objects in liquid suspension.

In previous studies on gold $^{7,8}$ and silver ${ }^{10}$ sols, a steady-state spectrum for each aggregation state was considered, which does not allow for extracting detailed information on the composition and optical properties of the aggregates. The method presented in the present work relies on chemometric analysis of a set of optical spectra (i.e. a spectrotemporal matrix) of a slowly aggregating suspension evolving over time, which does allow for the spectra of dimers to be identified and measured.

Two additional analytical techniques have been applied for the identification of nanoparticle dimers in the sample. The first technique is light scattering fluctuation correlation spectroscopy (LS-FCS) which analyses the composition of the colloidal mixture at the single particle level, and yields the diffusion coefficient of each species present. The second technique, charge-detection mass spectrometry (CD-MS), is used to analyse the mass distribution of the colloidal mixture at the level of individual particles in the megadalton to gigadalton mass range. ${ }^{33,34}$ In particular, the composition of complex colloidal mixtures can be determined using CD-MS, in good agreement with conventional TEM analyses, as was demonstrated recently. ${ }^{35,36}$ The advantage of both LS-FCS and CD-MS (which uses electrospray ionisation) is that these methods directly probe the composition of the liquid suspension at the time of measurement in contrast to electron microscopies where it is difficult to obtain samples that are representative of the composition of the sampled liquid.

\section{Materials and Methods}

\subsection{Aqueous suspensions of gold nanospheres}

Spherical citrate-stabilized gold nanoparticles, with an average diameter of $20 \mathrm{~nm}$, were synthesized following the Turkevich-Frens citrate-reduction method. ${ }^{37,38}$ Particles of 20, 50 and $80 \mathrm{~nm}$ diameter were obtained from Sigma-Aldrich (stabilized suspension in $0.1 \mathrm{mM}$ phosphate buffer, "reactant free") or British Biocell International (BBI, Cardiff, Wales, UK). The initial aqueous gold nanosphere (GNS) suspensions had concentrations corresponding to an optical density of approximately 1 at the maximum of the plasmon extinction band ('OD 1'). Particle concentrations were calculated from the optical densities measured at the maximum of the plasmon band, using extinction coefficients derived from the nominal 
particle diameter $(d)$, according to a simple, experimentally verified relation (Eqn. 1) based on Mie theory. ${ }^{22}$

$$
\varepsilon_{\max }=A(d / \mathrm{nm})^{\gamma}
$$

For gold nanospheres of diameters smaller than $85 \mathrm{~nm}$ in water, $A=4.7 \times 10^{4} \mathrm{M}^{-1} \mathrm{~cm}^{-1}$ and $\gamma=$ 3.30 .

\subsection{Functionalisation of the colloids with lipoic acid}

Racemic $( \pm)$ - $\alpha$-lipoic acid $(1 \mathrm{mM})$ and $\mathrm{NaOH}(2 \mathrm{mM})$ were dissolved in water to yield an aqueous solution of sodium lipoate (NaLA) at $\mathrm{pH}$ 11. This LA solution was added to the initial GNS suspensions. The amount of NaLA solution was chosen such so that the concentration of LA was twice the estimated concentration of binding sites on the GNS. This concentration was estimated by multiplying the particle concentration with the number of potential thiol binding sites $N_{\text {sites }}$ calculated ${ }^{25}$ using a semi-empirical formula, Eqn. 2.

$$
N_{\text {sites }}=77.9\left(\frac{(d / \mathrm{nm})}{2}\right)^{2}
$$

The mixtures were then left in the dark overnight. Subsequently, the samples were purified by three centrifugation-redispersion cycles in order to eliminate residual reactants, excess of citrate ions, ligand, and other stabilizers. Typically, the samples were centrifuged for $30 \mathrm{~min}$., at accelerations ranging from $4500 \mathrm{x} g$ for $20 \mathrm{~nm}$ GNS to $600 \mathrm{x} g$ for $80 \mathrm{~nm}$. The supernatant was removed and replaced by pure $1 \mathrm{mM}$ aqueous $\mathrm{NaOH}$. Typically, in each run $295 \%$ of the liquid volume was replaced by fresh aqueous $\mathrm{NaOH}$, removing most of the remaining excess ligands and salts. The final aqueous stock solutions used in the aggregation experiments contained LA-coated gold nanospheres (LA-AuNS) at a concentration corresponding to OD 1 in $1 \mathrm{mM} \mathrm{NaOH}(\mathrm{aq})$. In a separate series of preparations, the $1 \mathrm{mM} \mathrm{NaOH}(\mathrm{aq})$ used in the washing procedure contained additional NaLA at concentration varying from $1 \mu \mathrm{M}$ to $1 \mathrm{mM}$. The suspensions prepared in this manner thus contain an excess of NaLA.

\subsection{Optical spectroscopy}

Optical spectroscopy was carried out at ambient temperature (296 ( \pm 2$)$ K) on air-equilibrated samples contained in standard $1 \mathrm{~cm}$ quartz or plastic fluorescence cuvettes. UV-visible absorption spectra were measured using an optical fiber-based system (Ocean Optics) incorporating a USB4000-VIS-NIR CCD spectrometer and a LS-1 tungsten halogen light source $(6.5 \mathrm{~W}, 3100 \mathrm{~K})$ equipped with a BG34 colour correction filter. 
Resonant light scattering (RLS) measurements were performed according to the methodology that we described previously. ${ }^{22,39}$ The samples were illuminated with white light (LS-1 tungsten halogen light source with a BG34 colour correction filter), the scattered light was collected at a fixed angle of $90^{\circ}$, and analysed using a back-thinned CCD spectrograph (QE65000). A solvent blank background was subtracted from all spectra, and spectra were corrected using Ludox as the perfect Rayleigh scatterer. ${ }^{40}$ All samples were diluted such that the optical density remains well below 0.05 . The corrected light scattering spectra were obtained by calibration of the raw spectrum against the raw spectrum of Ludox, according to Equation 3.

$$
I_{\mathrm{LS}}(\lambda)=\lambda^{-4} \frac{I_{\mathrm{raw}}(\lambda)}{I_{\mathrm{raw}}^{\mathrm{Ludox}}(\lambda)}
$$

The Ludox sample consisted of the supernatant of a Ludox SM30 (Aldrich) suspension centrifuged for $1 \mathrm{~h}$ at $9700 \mathrm{x}$ g, diluted 200 times in $0.05 \mathrm{M} \mathrm{NaCl}$. For each measurement series, three Ludox reference samples were prepared, and each was characterised using its $p_{0}$ value, by fitting $\operatorname{OD}_{\text {Ludox }}(\lambda)=p_{0} \lambda^{-4}+p_{1}$ to the experimental Ludox extinction spectrum. The baseline value $p_{1}$ should be very close to zero, else a new reference sample is prepared. Finally, it was verified that all three Ludox samples gave identical scattering spectra, and the average of the $p_{0}$ was used to calculate the scattering cross sections (in $\mathrm{cm}^{2}$ ) from the corrected scattering spectra (with $c$ the concentration of particles in $\mathrm{mol} \mathrm{L}^{-1}$ ).

$$
\sigma_{\mathrm{sca}}(\lambda)=\frac{2303 p_{0}}{c N_{\mathrm{A}}} I_{\mathrm{LS}}(\lambda)
$$

\subsection{Method for extraction of the pure dimer spectrum from a sequence of optical spectra}

Optical spectra of particle dimers are obtained from suspensions of monomers undergoing slow and controlled aggregation. Aggregation of the suspended nanospheres was in general induced by adding a small volume of a concentrated $100 \mathrm{mM}$ or $200 \mathrm{mM} \mathrm{pH} 5.0$ acetate buffer to a suspension of LA-AuNS in $1 \mathrm{mM} \mathrm{NaOH}$. Final $\mathrm{pH}$ was calculated including the $1 \mathrm{mM}$ $\mathrm{NaOH}$, and verified using an ISFET pH meter. Ionic strength was calculated on basis of weak acid dissociation and ions present at the final suspension composition.

The time evolution of the optical spectra of the samples was recorded using the extinction or the scattering spectrometers. Each set of spectra obtained was analysed using a chemometric procedure. As the first step of this chemometric analysis, the series of spectra are represented 
as a numerical matrix, of which the columns contain the data points representing the timeevolution of the spectral response (optical density, scattering intensity) at a particular wavelength, and the rows the data points representing the optical spectrum at a particular time. By applying singular value decomposition (SVD) ${ }^{41,42}$ an indication of the number of species present is obtained, purely based on the information content of the matrix, without any supplementary (chemical) knowledge.

In the case of only two principal components, we use the following simple approach, which leads unambiguously to a realistic decomposition of the individual contributions after the optimization procedure for each dataset. It consists in subtracting a fraction of the reference monomer spectrum from each acquired spectrum. The temporal evolution of the fraction to be subtracted is governed by a kinetic equation. This kinetic equation can be purely empirical (single or multiple exponentials) or result from a kinetic model assuming a "bi-molecular" aggregation process. The basic idea behind the procedure is that after subtraction of the fraction of the monomer spectrum, the resulting candidate dimer spectrum should have a shape that does not change over time.

The optimization procedure searches for the parameters $\left(p_{0}, p_{1} \ldots p_{M}\right)$ of the kinetic model that minimize a cost function, which in this case is the wavelength-averaged standard deviation of the reconstructed dimer spectrum over time. It is a measure how much the estimated candidate dimer extinction spectrum varies over the set of reconstructed spectra using the kinetic model.

$$
\operatorname{cost}\left(p_{0}, p_{1} \ldots p_{M}\right)=\sum_{l=0}^{L} \sqrt{\frac{1}{N} \sum_{n=0}^{N}\left(d_{n l}-d_{l}^{\mathrm{avg}}\right)^{2}}
$$

$N$ is the number of time-steps for which a spectrum was acquired. $L$ is the number of measured wavelengths for each spectrum. $d_{n l}$ is the estimated extinction of the dimers for time step $n$ (corresponding to time $t_{n}$ ) and wavelength element $l$ (corresponding to wavelength $\lambda_{l}$ ),

$d_{l}^{\text {avg }}$ is the time average of the dimer response for the wavelength $\lambda_{l}: d_{l}^{\text {avg }}=\frac{1}{N} \sum_{n=0}^{N} d_{n l}$

The dimer spectra $d_{n l}$ are calculated according to Equation 6. 


$$
d_{n l}=\frac{s_{n l}^{\exp }-m_{l} c_{n}^{(m)}}{c_{n}^{(d)}}
$$

where, for each time step $n, s^{\exp }$ represents the measured spectra, $m_{l}$ is the reference monomer spectrum, $c^{(m)}$ is the time evolution of the concentration of monomers, $c^{(d)}$ is the corresponding time evolution of the dimer concentration. The concentrations at each time step $n$ are calculated from the kinetic model using parameters $\left(p_{0}, p_{1} \ldots p_{M}\right)$.

$$
\left\{\begin{array}{l}
c_{n}^{(m)}=f\left(t_{n}, p_{0}, p_{1} \ldots p_{M}\right) \\
c_{n}^{(d)}=g\left(t_{n}, p_{0}, p_{1} \ldots p_{M}\right)
\end{array}\right.
$$

The values of functions $f$ and $g$ either come from an analytical expression describing the concentration kinetics or evaluated numerically from differential equations. The chemometric procedures were implemented in the Python programming language (Python 2.7), with the numpy (version 1.8.1) and scipy (0.14.0) modules. In certain cases (spectra taken at low concentrations), a Savitsky-Golay filter was applied to the spectral data in order to obtain a smoother extracted experimental spectral profile for comparison with theoretical spectra.

\subsection{Light scattering fluctuation correlation spectroscopy}

The setup for light-scattering fluctuation spectroscopy (LS-FCS), which has previously been described $^{43}$ in detail, is built around an inverted microscope. The excitation is provided by a tunable $(700 \mathrm{~nm}-1000 \mathrm{~nm})$ femtosecond laser with a repetition rate of $80 \mathrm{MHz}$ and a pulse width of 100 fs. If necessary, the excitation light is frequency-doubled using a $\beta$-barium borate $(\mathrm{BBO})$ crystal. The laser beam is focused by a water-immersion objective (NA=1.2, 60x Nikon). A $25 \mu \mathrm{m}$ pinhole was used for confocal filtering. The detection was done using a 50/50 beamsplitter and two avalanche photodiodes (APDs, SPCM-AQRH, Perkin Elmer). Cross-correlation of the photon counts between the two detectors allows for elimination of after-pulsing and to exploit the microsecond domain. Scattering is an elastic process, and therefore no optical filters were used in the detection channel. As a consequence, the laser power was set at a relatively low level ( $2 \mathrm{nW}$ on the sample) in order to avoid saturation of the APDs. Since no optical filter is used, there is a residual background due to the light scattered by the solvent and the light reflected at the interface between the glass coverslip and the solvent. This latter source of noise can be attenuated by confocal filtering by working 
at a distance of $30 \mu \mathrm{m}$ above the glass coverslip. The acquisition and processing of data, and the calculation of the correlation functions are performed by home-written software.

\subsection{Charge Detection Mass Spectrometry}

Experiments were performed on a custom-built charge detection mass spectrometer with an electrospray ionisation (ESI) source. This instrument is described in detail elsewhere. ${ }^{44}$ Gold nanospheres of $80 \mathrm{~nm}$ diameter were first investigated in their monomeric form by injecting an as-supplied BBI suspension (see Fig. S11, Supporting Info). Then an aqueous suspension of $80 \mathrm{~nm}$ AuLA in $1 \mathrm{mM} \mathrm{NaOH}$ was acidified with dilute $\mathrm{HCl}$ to reach a $\mathrm{pH}$ close to the $\mathrm{pK}_{\mathrm{a}}$ of lipoic acid (4.7) in order to induce dimerisation. As higher ionic strengths of the medium may be detrimental for the electrospray ionisation process, careful direct acidification of the suspension was applied instead of ionic-strength control. Dimerisation was monitored with UV-visible spectrometry. After two hours of dimerisation, the sample was injected into the CD-MS device. The samples were injected at flow rates of typically $150 \mu \mathrm{L} / \mathrm{h}$, and entered the electrospray chamber through a $0.1 \mathrm{~mm}$ internal diameter stainless steel capillary tube located inside the needle tip. Nitrogen drying gas was injected between the end cap and the transfer glass capillary and flew through a heater typically set at $200^{\circ} \mathrm{C}$. The vacuum interface was composed of a glass transfer capillary that passes the ions into the first stage of the vacuum system, an end cap, a skimmer between the first and second vacuum stages, an hexapole ion guide and an exit lens. The charge detection device was used in a single pass mode. The signal induced on the tube was picked up by a JFET transistor and was amplified by a low-noise, charge-sensitive preamplifier and then shaped and differentiated by a homebuilt amplifier. The signal was recorded with a waveform digitizer card that recorded the entire waveform for each ion passing through the detector tube at a sampling rate of $10 \mathrm{MHz}$. The data were transferred to a desk-top computer where they were analyzed to compute the charge and mass of each ion. Internal calibration in charge was performed using a test capacitor that allowed a known amount of charge to be pulsed onto the pick-up tube. In addition, an external calibration was performed using NIST traceable size standards (70, 100, $150,200,250,300,350,400,450$ and $500 \mathrm{~nm}$ polystyrene nanospheres supplied by Polysciences Europe $\mathrm{GmbH}$ ). Further details of the calibration can be found in the supporting information of Ref. ${ }^{33}$. Mass histograms are built from the collection of a statistically relevant number of single mass measurements for each sample (>2000 typically). 


\subsection{Theoretical modeling of extinction and scattering spectra}

The optical cross sections of the spherical 'monomer' particles were obtained from analytical Mie theory expressions, as described by Bohren and Huffman ${ }^{45}$. Numerical evaluation of the analytical expressions (which involves summing over series of Bessel functions) was done using the Python code reported in previous work. ${ }^{22}$ The dielectric function of gold was by Johnson and Christy, ${ }^{46}$ with corrections for limited mean free path for electrons in small particles. ${ }^{47}$

For the dimers, theoretical calculation of extinction and scattering cross sections as a function of wavelength, and interparticle gap, was performed using DDSCAT (version 7.3.0) which implements the discrete-dipole approximation (DDA). ${ }^{48} \mathrm{~A}$ plane wave of linearly polarised light was assumed. The dimers were represented by a large number of discrete dipoles: $N=6.7 \times 10^{4}(20 \mathrm{~nm}$ particle diameter $), N=1 \times 10^{6}(50 \mathrm{~nm})$, and $N=5.5 \times 10^{5}(80$ $\mathrm{nm})$. The number of dipoles was chosen such that the relation $|m| k d<1$ was amply satisfied (here, $m$ is the ratio of the refractive index of the particle to the refractive index of the medium, $k$ is the wavevector of the incident light and $d$ is the spacing between elementary dipoles). In fact, we used $|m| k d<0.02$ for 20 and $50 \mathrm{~nm}$ particle dimers and $|m| k d<0.04$ for $80 \mathrm{~nm}$ particle dimers.

An additional relation, $|m-1|<2$, is often evoked as a criterion for obtaining satisfactory accuracy in the calculations, in relation with the polarizability of the elementary dipoles close to the gap of the dimers. This criterion is difficult to satisfy for metals even if an increased number of dipoles is considered. ${ }^{49}$ However, it has been recently demonstrated that DDA can be still applied for metals if the filtered coupled dipoles (FCD) approach is used rather than the usual lattice dispersion relation (LDR). ${ }^{50}$ In the FCD approach, the electric field generated by each elementary dipole is 'smoothed' by filtering out its high spatial frequencies. This alleviates problems for wavelengths for which gold has a large refractive index. The FCD method contributes also to reduction of the computational time, ${ }^{49}$ which is further reduced by using the Fourier transformation-based solver in DDSCAT.

The extinction, absorption and scacttering cross-sections for non-polarised light were averaged over all orientations because of the random orientation of the dimers in the suspending liquid medium. It can be demonstrated ${ }^{45}$ that for this object with axial symmetry the orientational average of the cross sections of extinction, absorption, and scattering, assuming a linearly polarized incident light along y is exactly equal to 


$$
\sigma=\frac{1}{3}\left(\sigma_{x}+\sigma_{y}+\sigma_{z}\right)
$$

where the $x, y$ and $z$ subscripts represent orientation of the long axis of the dimer along one of the three axes of the orthogonal laboratorium frame. The reported theoretical cross sections are orientational averages calculated using this relation.

\section{Results and discussion}

\subsection{Analysis of time-series of optical spectra of an aggregating suspension}

The determination of the absolute extinction cross section spectra relies on the measurement of the evolution of the optical spectra of a sample of suspended nanospheres as it evolves in time from an unaggregated to a partially aggregated state. The sample, initially at equilibrium in a non-aggregated state, is submitted to a sudden change in physicochemical conditions (change in $\mathrm{pH}$ and/or in ionic strength). Spectra are recorded as the sample evolves towards a new equilibrium state, in which a fraction of nanospheres will have aggregated into dimers. The series of spectra obtained for each experiment are readily analysed using chemometric methods that decompose the series of spectra into the spectra of the individual components present, together with the time-evolution of the concentration of each component.

The analysis method is illustrated using the spectral data of a typical experiment. Figure 1 shows the time-evolution of the UV-visible extinction spectrum of an aqueous suspension of $20 \mathrm{~nm}$ lipoic-acid functionalised gold nanospheres. At $t=0$, a small volume of concentrated $\mathrm{pH} 5.0$ acetate buffer was added to the initial LA-functionalised AuNS suspension (1 mM $\mathrm{NaOH}(\mathrm{aq})$ ) to yield a suspension in $6 \mathrm{mM}$ acetate buffer at $\mathrm{pH} 5.1$ (this takes into account the $1 \mathrm{mM} \mathrm{NaOH}$ initially present). The combination of a $\mathrm{pH}$ near the $\mathrm{pK}_{\mathrm{a}}$ of lipoic acid with an increased ionic strength induces a slow, well-controlled aggregation leading to the formation of an aggregation-related new plasmon resonance near $600 \mathrm{~nm}$. For comparison, native citrate gold NS were found not to aggregate under the same conditions. 


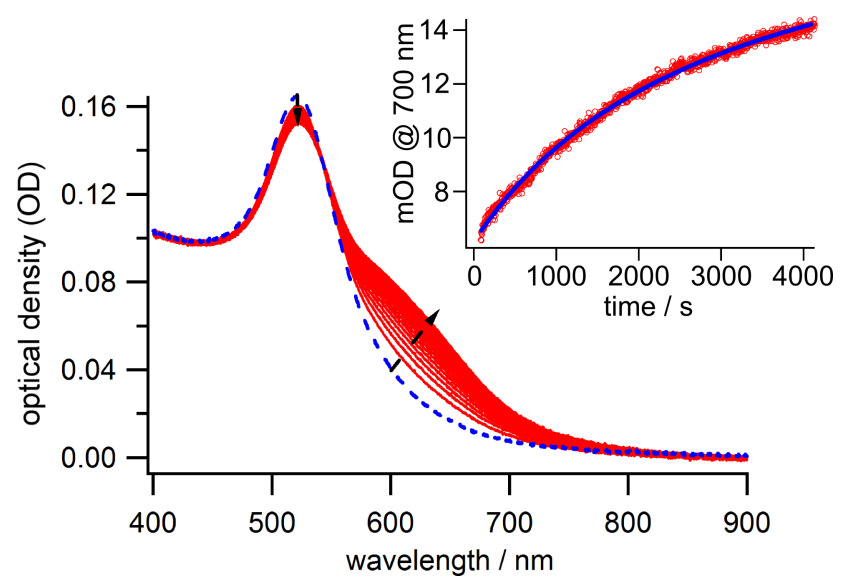

Figure 1. Evolution over 4000 seconds of the extinction spectrum of $20 \mathrm{~nm}$ gold nanospheres functionalised with LA in $6 \mathrm{mM} \mathrm{pH} 5.1$ acetate buffer (ionic strength $I=$ $\left.4.8 \times 10^{-3}\right)$. The blue dashed line represents the initial spectra of the $20 \mathrm{~nm}$ LA-AuNS prior to dimerisation. Inset: Time-evolution of the optical density at $700 \mathrm{~nm}$ (red circles). The blue line is a fit of Eqn. $9\left(c_{d}\right)$ to the data, with kinetic constant $k_{1}=4.4 \mathrm{x}$ $10^{-4} \mathrm{~s}^{-1}$. This constant serves as the initial guess in the iterative spectral decomposition procedure.

A distinct isosbestic point is observed around $545 \mathrm{~nm}$. An isosbestic point occurs in the case that a chemical species is converted into an other one in a reaction that has constant stoichiometry, ${ }^{51}$ and where initial species and the reaction products have distinct spectra. Here, the initial species are particle monomers, and for entropic reasons ${ }^{52}$ the product should necessarily be particle dimers. The isosbestic point survives only in the initial stages of the aggregation. Under the conditions used, this initial ("monomer-to-dimer conversion") stage lasts over more than an hour.

This interpretation is confirmed by the decomposition of the spectrotemporal matrix using singular-value decomposition (SVD, Fig. S1, Supporting Info), which indicates two principal components. For this particular dataset, that was chosen for illustrating our method and which spanned the first $4000 \mathrm{~s}$ of the aggregation process, we found that the quality of the data (i.e. "chemometric purity" of the two components) further improved when the first 500 seconds were not included in the analysis. This may be due to sub-optimal mixing conditions, as a small volume of a highly concentrated buffer solution is added to the initial volume, leading to a slight perturbation of the kinetics and the optical spectra in the first $500 \mathrm{~s}$. In 
further experiments, where milder mixing conditions were used (larger volumes of less concentrated buffers were added), this artifact was not present.

For the reconstruction of the individual component contributions to the overall spectrum using the method described in the Materials and Methods section, a kinetic model is needed that describes the evolution of the concentrations of nanoparticle monomers and dimers. We found that simple dimerisation kinetics (Eqn. 8) gives an accurate description of the initial stage of the slow aggregation process.

$$
2 A \stackrel{k_{\operatorname{dim}}}{\longrightarrow} A_{2}
$$

The evolution of concentration of monomers and dimers can be written analytically as Eqn. 9, where $c_{m}$ and $c_{d}$ are the concentrations of monomer and dimer, respectively, and kinetic constant $k_{1}=k_{\text {dim }} c_{\text {tot }}$, with $k_{\text {dim }}$ the bimolecular dimerisation rate constant, and $c_{\text {tot }}$ the initial concentration of monomer.

$$
\left\{\begin{array}{l}
c_{\mathrm{m}}(t)=\frac{c_{\mathrm{tot}}}{\left(1+k_{1} t\right)} \\
c_{\mathrm{d}}(t)=\frac{1}{2} \frac{c_{\mathrm{tot}} k_{1} t}{\left(1+k_{1} t\right)}
\end{array}\right.
$$

An initial confirmation that this model describes the experimental kinetics very well comes from a fit of the model to the evolution of the absorbance (Figure 1, inset) at a wavelength where the principal contribution comes from the dimer (e.g. $700 \mathrm{~nm}$ in Fig. 1, which is at the red edge of the dimer extinction, and far away from any monomer extinction). The value for $k_{1}$ obtained from this simple curve fit is used as an initial guess for the full analysis.

The total concentration of particles $c_{\text {tot }}$ is identical to $c_{m}$ at $t=0$. This initial concentration of monomer nanoparticles was calculated from the absorbance of the suspension using Eqn. 1, as described under 'Materials \& Methods'. The values of the absorbance spectrum of the monomer were furthermore scaled by dividing by $c_{\text {tot }}$ to represent the extinction coefficients, thus supplying the $m_{l}$ elements (see Materials \& Methods).

The optimisation finally relies on only one parameter, the kinetic constant $k_{1}$. The cost function displays a clear minimum, in this particular case for $k_{1}=4.4 \times 10^{-4} \mathrm{~s}^{-1}$. The optimisation yields the spectral and kinetic profiles corresponding to the monomer and the dimer (Fig. 2). We obtain absolute concentrations, and therefore also absolute cross sections. These cross sections are for the monomers and dimers randomly oriented in the liquid, and represent the orientational averages. 

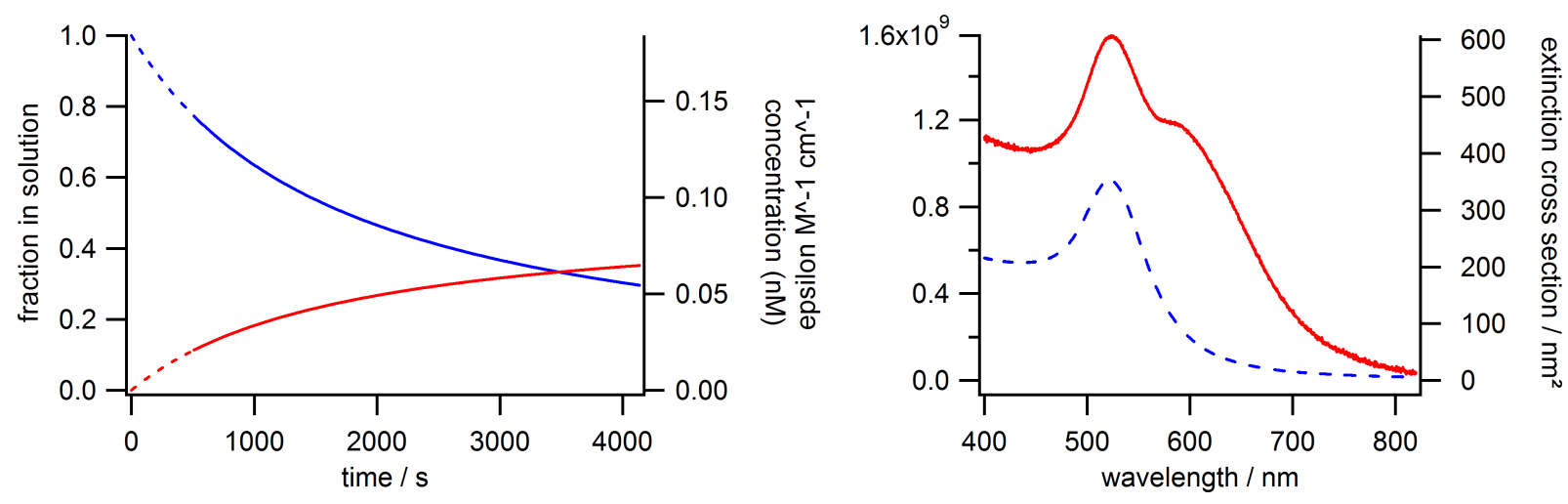

Figure 2. Left: Concentration evolution of the monomer (blue line) and the dimer (red line) obtained from the data in Fig. 1 by the data analysis procedure. Right: Extracted extinction spectra of the monomer (dashed blue) and dimer (solid red), considering the spectra recorded between 500 s and 4000s after the initiation of the aggregation.

The shape of the dimer extinction spectrum extracted from the series of spectra is in good agreement with numerical calculations (detailed below) as well as with spectra reported in the literature for dimers composed of gold particles of similar diameters. ${ }^{27,28}$ (see also Fig. S4, Supporting Info). The spectra in the literature do not give the absolute extinction coefficients, since the concentration of dimers after purification was not known. The chemometric approach used here yields absolute extinction coefficients, since the concentration of dimers at any stage in time is known, from the initial concentration of monomers, and the kinetic model.

\subsection{Identification of dimers at the single object level with fluctuation correlation spectroscopy}

Slowly aggregating suspensions of LA-AuNS were studied using light scattering fluctuation correlation spectroscopy (LS-FCS, also called SCS, scattering correlation spectroscopy ${ }^{53}$ ). LSFCS is similar to fluorescence correlation spectroscopy but uses the scattering of light by the nanoparticles instead of fluorescence. LS-FCS yields diffusional and rotational correlation times and is used here to confirm that the slowly aggregating suspensions contain practically only monomers and dimers. The dimers can be identified by their diffusion times. This diffusion time should be slightly less than twice the diffusion of the monomer. Additionally, whereas GNS monomers are isotropic and display no rotation-related intensity fluctuations in their scattered light, the dimers are anisotropic and do display intensity fluctuations due to 
Brownian rotation. The associated rotational correlation times can be used for further characterisation.

For LS-FCS we worked with $50 \mathrm{~nm}$ AuNS monomers. The aggregation of $50 \mathrm{~nm}$ LA-AuNS is triggered by adding concentrated $\mathrm{pH} 5.0$ acetate buffer to obtain a mixture of $9 \mathrm{mM}$ of buffer and $1 \mathrm{mM}$ of $\mathrm{NaOH}$ (yielding $\mathrm{pH} 5.1$, ionic strength $I=8.6 \times 10^{-3}$ ). The aggregation is then very slow and proceeds over a period of several hours. The experimental correlation curve is shown in Fig. 3. The correlation curve does not display bumps or other artifacts. The presence of large aggregates beyond dimers would give very strong scattering signals and lead to a correlation curve that would be difficult to analyse.

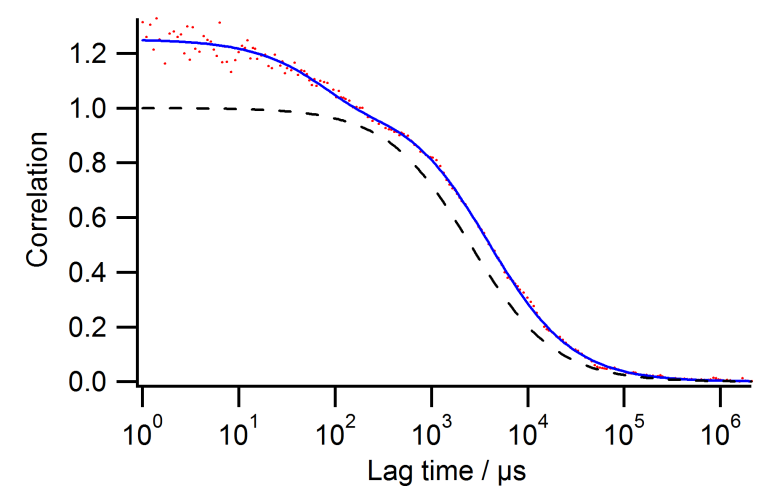

Figure 3. Normalized correlation curve (red dots) of light scattering signal of GNS dimers measured at $760 \mathrm{~nm}$. The fit (blue line) gives a diffusion time of $3.8 \mathrm{~ms}$ and a rotation time of $70 \mu \mathrm{s}$. Black dashed line: the predicted correlation curve of a monomeric $50 \mathrm{~nm}$ GNS at $760 \mathrm{~nm}$, obtained by adapting experimental data obtained at $520 \mathrm{~nm}$ (see Figure S10, Supporting Info)

The experimental correlation curve is well described by a theoretical curve corresponding to a single population with a diffusion time of $3.8 \mathrm{~ms}$ and a rotational component of $70 \mu \mathrm{s}$. At the wavelength used, $760 \mathrm{~nm}$, the scattering by dimers dominates the signal. The observed diffusion time is larger than the diffusion time of the monomer $(2.55 \mathrm{~ms})$, and in good agreement with the expectations for a dimer. The experimental ratio of the equivalent hydrodynamic radius of monomer and aggregates is 1.5 . The diffusion time of the monomer at $760 \mathrm{~nm}$ has been derived from a light-scattering FCS measurement made at $520 \mathrm{~nm}$ where the scattering of the monomer is resonant (the monomer does not respond at $760 \mathrm{~nm}$ ), and 
adapting this value to correct for the differences in focal volume between $520 \mathrm{~nm}$ and $760 \mathrm{~nm}$ (see Supporting Info, Figure S10).

The presence of a rotational contribution in the light-scattering FCS curve indicates their geometric anisotropy. Spheres do not display such a rotational contribution. The measured rotational diffusion time of the dimers is $70 \mu \mathrm{s}$. This experimental value lies between the theoretical value $(88 \mu \mathrm{s})$ obtained for a $20 \mathrm{~nm}$ x $40 \mathrm{~nm}$ rod using theory by Tirado et al. ${ }^{54}$ and the value $(56 \mu \mathrm{s})$ obtained for a dimer of spheres by simulation with the "hydro++" computer program. ${ }^{55}$ The rotational diffusion time depends on the volume of the nanoparticle. Hence, the measured value confirms the absence of aggregates beyond dimers, such as trimers that would have rotation times beyond $100 \mu \mathrm{s}$.

\subsection{Identification of dimers at the single-object level by charge-detection mass spectrometry}

Dimers of gold nanospheres in the aggregating suspensions were furthermore identified in charge-detection mass spectrometry (CD-MS). This MS-based technique appeared recently as a powerful analytical tool to study complex mixtures of very high-molecular weight components such as late intermediates in virus capsid assembly ${ }^{56}$ or composite clusters of nanoparticles. ${ }^{35,36}$ Here, CD-MS provides further proof of the existence of gold dimers. The experimental mass spectrum of freshly prepared dimers of $80 \mathrm{~nm}$ LA-AuNS is shown in Fig. 4. At a first glance and having in mind that $80 \mathrm{~nm}$ diameter gold nanospheres in their monomer state are characterized by a mean molecular weight of around 2.4 GDa (see Figure S11), the shape of the distribution is compatible with the presence of a majority of dimers. 


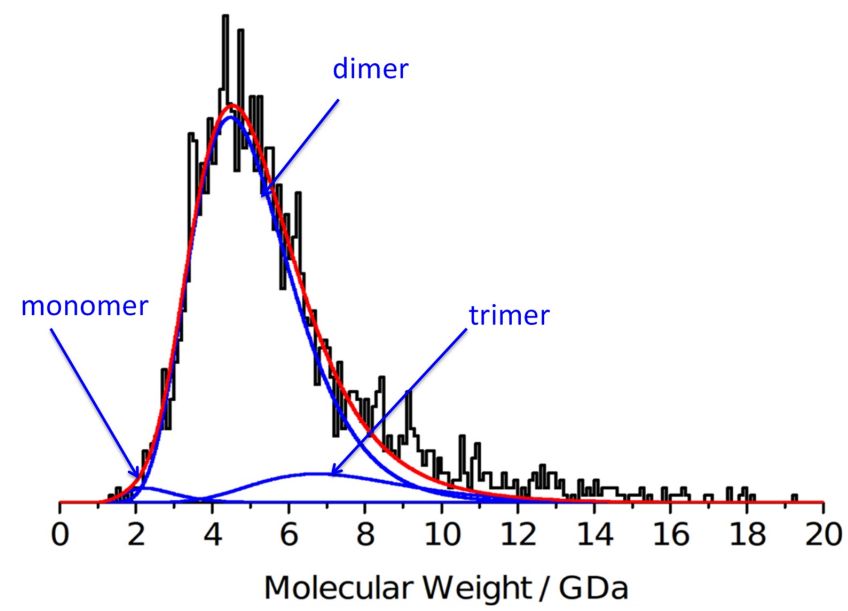

Figure 4. Experimental mass spectrum (black line) of freshly prepared $80 \mathrm{~nm} \mathrm{LA}$ AuNS dimers. Proposed combination (red line) of monomers, dimers and trimers populations (blue lines) that best fit the experimental mass distribution. Experimental mass spectrum of the monomer (see Fig. S11) was used as a basis for the analytical description (log-normal distribution) of monomer, dimer, and trimer populations.

Beyond this qualitative analysis, the mass spectrum can be further deconvolved as a combination of monomer, dimer, and trimer populations, the experimental mass distribution of the monomer serving as a basis. Once the weight of each population is adjusted to best fit the experimental distribution, it gives relative proportion of around $88 \%$ dimers (4.9 GDa), 10 $\%$ trimers $(7.3 \mathrm{GDa})$, and below $2 \%$ monomers. This distribution represents the aggregation state of the (evolving) suspension at the time that it is injected into the electrospray source. It corresponds to an aggregating suspension that has already evolved to the point that trimers are being formed from the dimers present. The electrospray ionisation required conditions of low ionic strength, high acidity for the aggregation, allowing less precise control of the aggregation process, in contrast to the optical spectroscopic measurements where the data acquisition was stopped before any observable trimers were formed (as evidenced from the persistence of the isosbestic point and two principal components in singular value decomposition). No further mass spectrometric monitoring or control of the kinetics was attempted at this stage. 


\subsection{Physicochemical behaviour of the aqueous colloidal gold - lipoic acid system}

\subsubsection{DLVO-based modeling}

The stability and aggregation of colloidal suspensions can be considered in the light of DLVO theory. ${ }^{52,57}$ Recently, this theory has been applied to the study of the kinetics of (irreversible) aggregation of gold nanoparticles induced by ligand exchange. ${ }^{8}$ In the DLVO model, the potential energy of the interaction of two colloidal particles of the same type is composed of a repulsive electrostatic term and an attractive Van der Waals term.

$$
V_{\mathrm{DLVO}}=V_{\text {elec }}+V_{\mathrm{VdW}}
$$

The electrostatic term is related to the surface charge and the electric double layer of each particle. The typical length scale of the electrostatic interaction is represented by the Debye length ${ }^{57} r_{\mathrm{D}}$ and its inverse $\kappa$, according to Equation 11 (see Appendix for definition of symbols).

$$
r_{\mathrm{D}}=\kappa^{-1}=\sqrt{\frac{\varepsilon_{0} \varepsilon_{\mathrm{r}} k_{\mathrm{B}} T}{2 \rho e^{2} N_{\mathrm{A}} I b^{\varnothing}}}
$$

The ionic strength is calculated from the molality of the dissolved ions and their charge numbers. In our experiments, the Debye length typically varies between $9.6 \mathrm{~nm}\left(I=1.0 \times 10^{-3}\right)$ and $3.0 \mathrm{~nm}\left(I=1.0 \times 10^{-2}\right.$, e.g. $15 \mathrm{mM}$ acetate buffer at $\left.\mathrm{pH} 5.1\right)$.

The potential for the electrostatic interaction is approximated by one of two expressions, depending on the interparticle separation with respect to the Debye length. When $s / r_{\mathrm{D}}<5$, we have

$$
V_{\text {elec }}=4 \pi \varepsilon_{0} \varepsilon_{\mathrm{r}} a^{2} Y^{2}\left(\frac{k_{\mathrm{B}} T}{e}\right) \frac{\exp \left(-s / r_{\mathrm{D}}\right)}{s+2 a}
$$

where

$$
Y=\frac{8 \tanh \left(\varepsilon_{0} \varepsilon_{\mathrm{r}} \psi_{0} / 4 k_{\mathrm{B}} T\right)}{1+\left[-2 \kappa a /(\kappa a+1)^{2} \tanh ^{2}\left(\varepsilon_{0} \varepsilon_{\mathrm{r}} \psi_{0} / 4 k_{\mathrm{B}} T\right)\right]^{1 / 2}}
$$

When $s / r_{\mathrm{D}}>5$, we have

$$
V_{\text {elec }}=2 \pi \varepsilon_{0} \varepsilon_{\mathrm{r}} \psi_{0}^{2} a \ln \left[1+\exp \left(-s / r_{\mathrm{D}}\right)\right]
$$

The attractive Van der Waals interaction between two particles can be described using the following potential: 


$$
V_{\mathrm{VdW}}=-\frac{A_{\mathrm{H}}}{6}\left[\frac{2 a^{2}}{R^{2}-4 a^{2}}+\frac{2 a^{2}}{R^{2}}+\ln \frac{R^{2}-4 a^{2}}{R^{2}}\right]
$$

This potential can be reliably approximated ${ }^{58}$ by Eqn. 16. A Hamaker constant $A_{\mathrm{H}}$ of $2.5 \times 10^{-19}$ J for gold was taken. ${ }^{8}$

$$
V_{\mathrm{VdW}}=-\frac{A_{\mathrm{H}}}{12} \frac{a}{s}
$$

However, the DLVO model of Eqn. 10 does not explain the fact that the aggregation state of suspensions of LA-coated gold colloids can reversibly be switched by changing the $\mathrm{pH}$ of the suspension, ${ }^{22}$ and that the individual colloidal particles in the aggregates thus should retain their integrity instead of becoming glued together. To account for this, a repulsive term is added to the DLVO potential that dominates at very short interparticle surface-to-surface distances. This term accounts for the steric stabilisation by the lipoic acid ligand shell surrounding the particles, as indicated in Eqn. 17.

$$
V_{\text {tot }}=V_{\text {elec }}+V_{\mathrm{VdW}}+V_{\text {steric }}
$$

We chose a Lennard-Jones style repulsive term (Eqn. 18), with adjustable parameters $K_{\mathrm{LJ}}$ corresponding to the strength of the steric repulsion, and $s_{\mathrm{m}}$, determining the thickness of the molecular layer around the particle.

$$
V_{\text {steric }}=K_{\mathrm{LJ}}\left(\frac{s_{\mathrm{m}}}{s}\right)^{12}
$$

In absence of more precise knowledge, values for $K_{\mathrm{LJ}}$ and $s_{\mathrm{m}}$ were chosen $\left(10^{-21} \mathrm{~J}\right.$ and $1.5 \mathrm{~nm}$, respectively) such that the final potential energy curves generated were in qualitatitve agreement with: (1) the observation of reversible aggregation of the AuLA nanoparticles when switching between low and high $\mathrm{pH}$, (2) the observation of control of the aggregation by changing the ionic strength at a $\mathrm{pH}$ near the pKa of lipoic acid, and (3) an interparticle distance (shortest surface-to-surface distance) on the order of $1 \mathrm{~nm}$.

With this set of parameters, the potential energy curve (Eqn. 17) does indeed reflect the observed reversible aggregation of the particles under influence of $\mathrm{pH}$ (see Fig. 5). At high $\mathrm{pH}$ (i.e. $\mathrm{pH}>>\mathrm{pK}_{\mathrm{a}}$ ), we assume a highly negative surface potential of $\psi_{0}=-120 \mathrm{mV}$ in order to account for the negative charges of the deprotonated lipoate ligands. This generates a repulsive curve, according to which the colloidal suspension is stabilised in an unaggregated state. Switching to low $\mathrm{pH} \ll \mathrm{pK}_{\mathrm{a}}$, the neutralisation of lipoate anions via protonation is modeled by setting the potential to $\psi_{0}=-30 \mathrm{mV}$ or even less negative. This yields a curve 
whose minimum corresponds to a dimer of particles with an interparticle separation of $\sim 1 \mathrm{~nm}$. This distance, which results directly from our guesses for the values of $s_{\mathrm{m}}$ and $K_{\mathrm{LJ}}$, is roughly in line with the interparticle distance suggested from the position of the longitudinal plasmon band in DDA modeling (see below). Note that between the two calculated curves, only the surface potential was varied; other parameters (such as ionic strength) were kept constant.

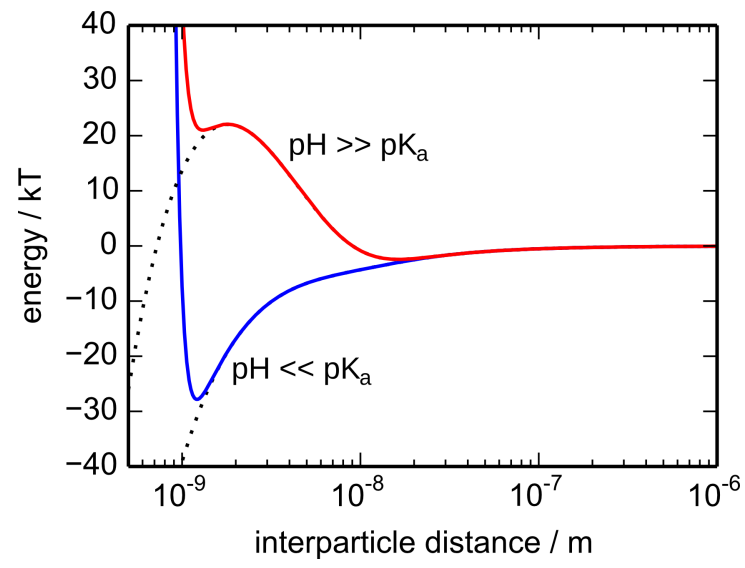

Figure 5. Two-particle potential energy curves according to Eqn 17, for $20 \mathrm{~nm}$ diameter gold nanoparticles in water at a constant ionic strength of $I=6.4 \times 10^{-3}$. For $\mathrm{pH}>>\mathrm{pK}_{\mathrm{a}}$ a surface potential $\psi_{0}=-120 \mathrm{mV}$ was taken, for $\mathrm{pH}<<\mathrm{pK}_{\mathrm{a}} \psi_{0}=-30 \mathrm{mV}$. $\mathrm{T}=$ $298.15 \mathrm{~K}, \mathrm{k}_{\mathrm{B}} \mathrm{T}=4.12 \times 10^{-21} \mathrm{~J}$. The dotted curves present the standard DLVO potential, without the steric term.

The present DLVO-based model also shows how the aggregation rate can be controlled by varying the ionic strength at a constant $\mathrm{pH}$ just above $\mathrm{pK}_{\mathrm{a}}$ using a monovalent (acetate) buffer at different buffer concentrations. The condition of a $\mathrm{pH}$ just above $\mathrm{pK}_{\mathrm{a}}$ was modeled by setting the surface potential to an intermediate value, $\psi_{0}=-65 \mathrm{mV}$. The two-particle potential energy curves at this intermediate surface potential display a diminishing barrier to aggregation on going from ionic strength $I=0.001$ to $I>0.008$ (Fig. 6). Near $I=0.006$, a small remaining barrier $\left(\sim\right.$ several $\left.k_{B} T\right)$ is consistent with slow ("reaction-limited") aggregation. 


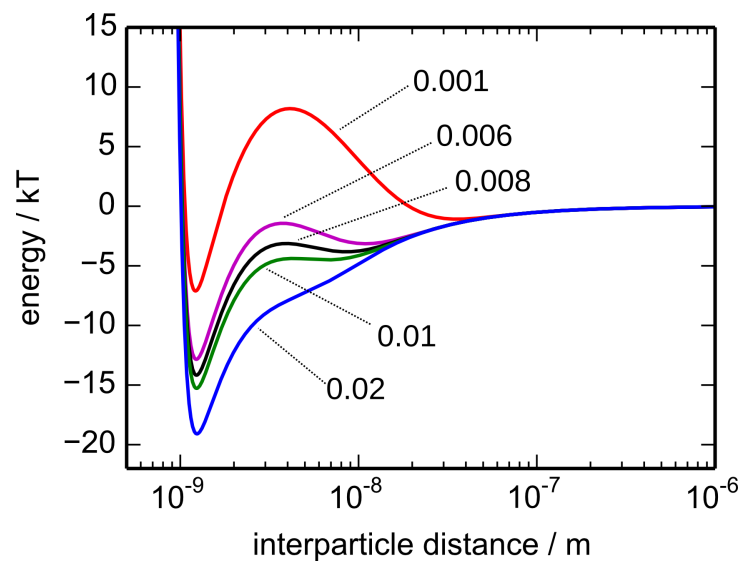

Figure 6. Two-particle potential energy curves (Eqn. 17) as a function of ionic strength, for $20 \mathrm{~nm}$ diameter gold nanoparticles in water, at intermediate surface potential $\left(\psi_{0}=-65 \mathrm{mV}\right)$, which was taken to model $\mathrm{pH} \approx \mathrm{pKa} . T=298.15 \mathrm{~K}, k_{\mathrm{B}} T=4.12$ $\mathrm{x} 10^{-21} \mathrm{~J}$.

Figure 6 further suggests that under certain circumstances the system may exist with a double-well potential, which would translate into dimers that thermally switch between two distinct interparticle distances. Barriers for switching between the two states depend on parameters such as the relative strength of the steric repulsion $K_{\mathrm{LJ}}$ and the surface potential $\psi_{0}$ to which we have no direct access. We used realistic, but estimated surface potentials.

While the DLVO model has been reported to have limitations, ${ }^{59,60}$ it does give a plausible, and semi-quantitative, explanation of the experimental observations. It also suggests the interest of a more detailed study of aggregation rates, and their dependence on temperature, ionic strength and $\mathrm{pH}$ for further control of particle dimerisation. For instance, controlled particle dimerisation is crucial for building up nanostructures using DNA-modified nanoparticles,${ }^{29}$ and rational control of interparticle forces may aid successful self-assembly.

3.4.2 Effects of $\mathrm{pH}$, ionic strength and ligand concentration. Comparison with experiments In the aqueous lipoic acid/gold nanosphere (LA-AuNS) system, decreasing the $\mathrm{pH}$ of the solution below the $\mathrm{pK}_{\mathrm{a}}$ leads to dimerisation of the gold nanospheres, rapidly followed by the formation of larger aggregates. As explained above, at decreasing $\mathrm{pH}$, protonation of the lipoate anion at the surface of the AuNS leads to a less negative surface potential, and the disappearance of the energetic barrier against nanosphere aggregation

The $\mathrm{pK}_{\mathrm{a}}$ of lipoic acid in aqueous solution has been reported between 4.76 and 5.3. ${ }^{61}$ Its $\mathrm{pK}_{\mathrm{a}}$ has been determined to be 5.6 in a self-assembled monolayer on the surface of $15 \mathrm{~nm}$ gold 
nanospheres deposited onto a silica substrate. ${ }^{62}$ For these particles, the surface potential was estimated to be $-173 \mathrm{mV}$ with respect to zero in the bulk of the solution, at high $\mathrm{pH}$ with all lipoic acid groups deprotonated.

We determined the initial rates of dimerisation from the kinetic profiles obtained from extinction spectroscopy for various values of $\mathrm{pH}$, while keeping the ionic strength approximately constant. There is a sudden and steep increase in the aggregation rate for $\mathrm{pH}$ $<4.9$ (see Figure S2, Supporting Info). For $20 \mathrm{~nm}$ NS below pH 5, the aggregation becomes difficult to control, progressing quickly beyond dimerisation.

Control of the dimerisation using solely the $\mathrm{pH}$ is not very precise. As suggested by the analysis of the system using DLVO theory, the aggregation rate can be "fine-tuned" by varying the ionic strength while keeping the $\mathrm{pH}$ constant. One way to achieve this is by changing the buffer concentration (see Figure S3, Supporting Info) as demonstrated. Alternatively, salt (e.g. $\mathrm{NaCl}$ ) may be added to create a saline buffer, which is the preferred method, since the ionic strength of a non-saline buffer at constant $\mathrm{pH}$ increases sublinearily as a function of buffer concentration.

The ionic strength of the medium provides a precise way of controlling the dimerisation kinetics. We found, however, that also the optical spectrum of the dimer is affected by the ionic strength. This is illustrated by Fig. 7, which shows how the extinction spectrum of dimers of $20 \mathrm{~nm}$ diameter AuLA changes as the acetate buffer concentration is changed. These dimer spectra were obtained using the chemometric method from the sets of optical spectra of the aggregating suspensions, under conditions where only two principal components appear in SVD, and a clear isosbestic point exists. 


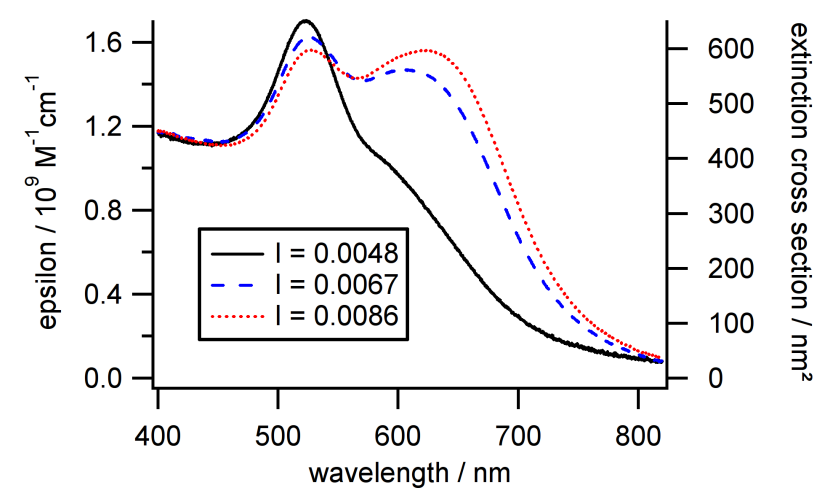

Figure 7. Absolute extinction spectra determined for dimers of $20 \mathrm{~nm}$ LA-AuNS formed at $\mathrm{pH} 5.1$ at different ionic strengths.

The red shift and increasing intensity of the long-wavelength longitudinal plasmon indicates that the plasmonic coupling between the particles in the dimer becomes stronger at higher ionic strength. Similar observations of ionic-strength dependence of plasmonic coupling have been made in gold nanoparticle dimers constructed using DNA. ${ }^{15}$ For most experiments in this work, we used low ionic strength conditions, and indicate the ionic strength used where necessary.

The experiments reported here were carried out using LA-AuNS in aqueous solutions containing just the amount of lipoic acid that is associated with the functionalisation of the particle surface. Interestingly, we found that when excess lipoic acid is added, both the dimerisation kinetics and the dimer extinction spectrum are affected. For instance, the presence of $10 \mu \mathrm{M}$ of LA prevents $20 \mathrm{~nm}$ GNS from dimerising in $6 \mathrm{mM}$ acetate buffer. Aggregation can still be triggered by going to higher ionic strengths or to a lower $\mathrm{pH}$. At 1 $\mathrm{mM}$ of LA, aggregation only starts significantly below $\mathrm{pH} 4$. The aggregation was therefore induced by adding $2.5 \mathrm{mM} \mathrm{HCl}$ to the solution, bringing the $\mathrm{pH}$ to 3.3 .

In addition to providing a greater barrier against aggregation, an excess of lipoic acid also leads to a dimer spectrum that is different from the dimer spectrum without additional lipoic acid (Fig. 8). The dimer extinction spectra (obtained chemometrically from sets of spectra of slowly aggregation suspensions, under conditions where only two principal components appear in SVD) show only a single, broad resonance when higher concentrations of lipoic acid are present, which indicates that the longer-wavelength longitudinal plasmon resonance is blue-shifted compared to dimers formed at low LA concentration, and that it merges into 
the shorter-wavelength transverse resonance as an almost indescernable shoulder into the shorter-wavelength transverse resonance.

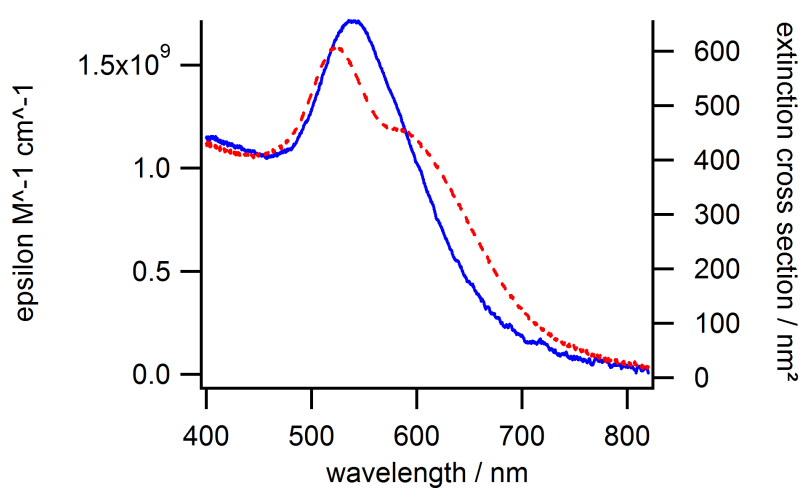

Figure 8. Spectra of $20 \mathrm{~nm}$ LA-AuNS dimers formed (a) without additional LA, (pH 5.1, $I=4.8 \times 10^{-3}$, red dashed curve $)$ and (b) excess LA ( $1 \mathrm{mM} \mathrm{LA}, \mathrm{pH} 3.3, I=2.5 \times 10^{-3}$, solid blue curve)

This blue shift of the longitudinal dimer resonance indicates a larger separation between the two GNS composing the dimer. ${ }^{63}$ The position of the maximum of absorbance of the dimer is very sensitive to the separation length of the dimer. The plasmonic shift $\Delta \lambda$ due to the interaction between the two GNS is empirically described ${ }^{63}$ by:

$$
\frac{\Delta \lambda}{\lambda_{0}}=a \exp \left(\frac{-s}{f D}\right)
$$

where $\frac{\Delta \lambda}{\lambda_{0}}$ is the fractional plasmon shift, $s$ is the interparticle edge-to-edge separation, $D$ the monomer diameter. $f$ is a coefficient so that $f D$ gives the characteristic distance of the interaction. This coefficient $f$ is almost independent of the monomer size or shape and is close to 0.2 for gold, $a$ is the plasmon shift amplitude which depends on the monomer diameter and the dielectric constant of the medium. In the present case, we do not have access to the absolute value of the interparticle distance since we have no knowledge of $a$.

The difference of interparticle distances $\Delta s$ between the case without free LA ("minLA") and the case with an excess of LA ("xsLA") can be calculated as

$$
\Delta s=\ln \left(\frac{\Delta \lambda_{\operatorname{minLA}}}{\Delta \lambda_{\mathrm{xsLA}}}\right) f D
$$


Taking 0.2 for $f$, we find that the monomers in dimers formed with an excess of LA are $1.5 \mathrm{~nm}$ closer than without the excess. This effect may be explained by assuming that particles in solutions not containing an additional ('excess') amount of LA are covered by less than a full monolayer of extended LA molecules. Indeed, such a partial coverage would allow the monomer to be closer during the aggregation process. At high free LA concentration (more than several $\mu \mathrm{M}$ ), the GNS surface is saturated and steric effects prevent the monomers from approaching, resulting in a dimer spectrum that is blue-shifted with respect to the samples that do not contain an excess of LA.

\subsection{Extinction cross sections of gold nanosphere dimers in water}

The physicochemical conditions for slow dimerisation of GNS having been established, the absolute, orientationally averaged extinction spectra of dimers of GNS of various diameters were determined from sets of extinction spectra evolving over time, using the methodology described above. The data sets concerning the evolution of the extinction spectra for 50 and $80 \mathrm{~nm}$ LA-AuNS can be found in the Supporting Information (Figures S6 and S7), the data for $20 \mathrm{~nm}$ LA-AuNS is found in Figure 1. In all cases, the extracted extinction spectrum of the dimer contains two plasmonic resonances (Figure 9). The resonance at shorter wavelengths (maximum between 520 and $580 \mathrm{~nm}$ ) is the so-called transverse resonance (i.e. polarised perpendicularly to the long axis of the dimer). The longer-wavelength resonance is the longitudinal resonance which has its transition dipole oriented along the long axis of the dimer. 

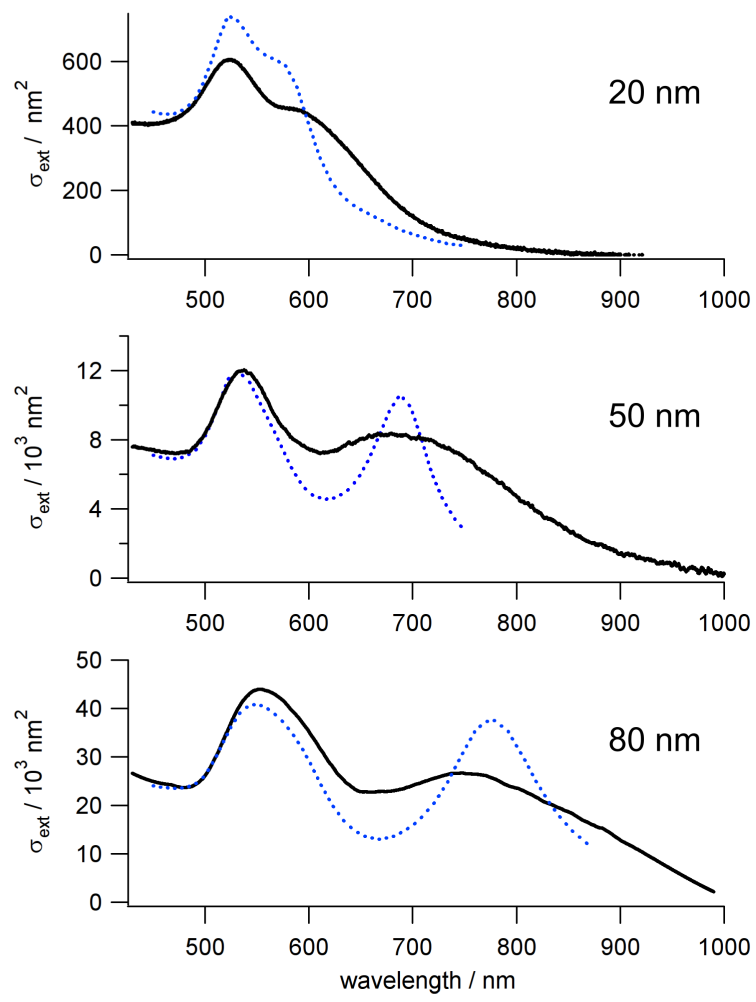

Figure 9. Absolute extinction cross section spectra of dimers of $20 \mathrm{~nm}, 50 \mathrm{~nm}$ and 80 nm gold nanospheres in water, obtained chemometrically from sets of extinction spectra of slowly aggregating suspensions evolving in time (solid black lines). Blue dotted lines: numerically calculated absolute extinction cross section spectra (dipoledipole approximation, see below) for idealised dimers in water, with a fixed interparticle gap of $1 \mathrm{~nm}$.

The longitudinal resonance of the dimers shifts to longer wavelength for larger particle diameters. As we already mentioned, the spectra of the $20 \mathrm{~nm}$ dimers are in good agreement with spectra reported in the literature for dimers composed of gold particles of similar diameters. $^{26,27}$ (see also Fig. S4, Supporting Info). For dimers of $50 \mathrm{~nm}$ and $80 \mathrm{~nm}$ no comparable spectra have been reported. Most importantly, here we report absolute extinction cross sections, which can be used for quantitative comparisons, calculation of dimer concentrations, and prediction of optical properties for dimer-doped materials. Using the method explained here these absolute cross sections are obtained without chemically separating the dimers from the monomers.

Table 1 collects the positions of the resonance maxima for different particle dimers. For the 
$20 \mathrm{~nm}$ dimers, the results at different ionic strengths are included (see Figure 7), as a reminder of the sensitivity towards this experimental parameter. For $50 \mathrm{~nm}$ and $80 \mathrm{~nm}$ the effect of varying ionic strength was not studied. These particles aggregate more slowly. Lower $\mathrm{pH}$ and/or higher ionic strength were used to induce aggregation for these particles.

Table 1. Light extinction by gold nanosphere dimers: wavelength maxima and extinction cross sections for both the transverse ("green") and longitudinal ("red") resonances.

\begin{tabular}{lcccccccc}
\hline & & \multicolumn{3}{c}{ transverse resonance } & \multicolumn{3}{c}{ longitudinal resonance } \\
$\begin{array}{l}\text { NS diam. } \\
(\mathrm{nm})\end{array}$ & $\mathrm{pH}$ & $\begin{array}{c}\text { ionic } \\
\text { strength }\end{array}$ & $\begin{array}{c}\lambda_{\max } \\
(\mathrm{nm})\end{array}$ & $\sigma_{\text {ext }}\left(\mathrm{nm}^{2}\right)$ & $\varepsilon\left(\mathrm{M}^{-1} \mathrm{~cm}^{-1}\right)$ & $\lambda_{\max }$ & $\sigma_{\text {ext }}\left(\mathrm{nm}^{2}\right)$ & $\varepsilon\left(\mathrm{M}^{-1} \mathrm{~cm}^{-1}\right)$ \\
\hline $20 \mathrm{~nm}$ & 5.1 & $4.8 \times 10^{-3}$ & 524 & $6.0 \times 10^{2}$ & $1.6 \times 10^{9}$ & 590 & $4.4 \times 10^{2}$ & $1.2 \times 10^{9}$ \\
& 5.1 & $6.7 \times 10^{-3}$ & 525 & $6.2 \times 10^{2}$ & $1.6 \times 10^{9}$ & 600 & $6.1 \times 10^{2}$ & $1.6 \times 10^{9}$ \\
& 5.1 & $8.6 \times 10^{-3}$ & 526 & $6.0 \times 10^{2}$ & $1.6 \times 10^{9}$ & 630 & $6.0 \times 10^{2}$ & $1.5 \times 10^{9}$ \\
$50 \mathrm{~nm}$ & 3.6 & $1.1 \times 10^{-3}$ & 535 & $1.2 \times 10^{4}$ & $3.1 \times 10^{10}$ & 690 & $8.2 \times 10^{3}$ & $2.1 \times 10^{10}$ \\
$80 \mathrm{~nm}$ & 5.0 & $2.7 \times 10^{-2}$ & 552 & $4.4 \times 10^{4}$ & $1.1 \times 10^{11}$ & 745 & $2.7 \times 10^{4}$ & $6.9 \times 10^{10}$
\end{tabular}

As an illustration of the strength of the optical response of plasmonic nanoparticles and their aggregates, it is interesting to compare the optical cross sections determined here to the physical dimensions of the particles. For plasmonic particles, the optical cross sections can be significantly larger than their physical cross sections, demonstrating that these small conducting structures indeed act as antennae at optical frequencies. The ratio of the optical and physical cross sections is given by $Q=\sigma_{\text {optical }} / \sigma_{\text {physical. }}$ These relative cross sections $Q$ are calculated for the total extinction, the absorption and the scattering cross sections. They are sometimes referred to as "Mie efficiencies", since the results of calculations of optical cross sections using Mie theory ${ }^{45}$ are typically expressed in terms of these dimensionless numbers.

The physical cross section of spherical particles is the area of a circle with the same radius as the sphere $\left(\sigma_{\text {physical }}=\pi a^{2}\right)$. For anisometric objects, such as particle dimers, it is less trivial to define an effective physical cross section, since the physical cross section the object presents depends on its orientation with respect to the incoming light. We use here the convention that the physical cross section of a dimer corresponds to that of an effective 
sphere that occupies the same volume as the dimer $\left(\sigma_{\text {physical }}=2^{2 / 3} \pi a^{2}\right)$. This convention, which is also used by the DDSCAT ${ }^{48}$ code, gives relative cross sections that are smaller than simply the sum of the cross sections of each constituent particle. This other convention ( $\sigma_{\text {physical }}=2 \pi a^{2}$ ) is used for exemple by Khlebtsov et al. ${ }^{64}$ and gives values for the relative cross sections that are systematically $26 \%$ lower than the "effective sphere" values. Obviously, these relative values can be readily interconverted, and the absolute optical cross sections remain unaffected by the choice of physical cross section convention.

The relative cross sections ('Mie efficiencies') for overall light extinction by monomers and dimers are collected in Table 2. For the monomers, the values are obtained from analytical Mie calculations, numerically evaluated. These have previously been shown to be in excellent agreement with experimental values. ${ }^{22,47,65,66}$ These relative cross sections significantly exceed unity, in particular for the larger particles, demonstrating the strength of their interaction with light at the plasmon resonance. For comparison, the $Q_{\text {ext }}$ values for molecular dyes are without exception inferior to 0.1 , even for potent light absorbers such as porphyrins (their Soret bands having extinction cross sections up to $0.15 \mathrm{~nm}^{2}$ for a molecular radius of approximately $9 \AA$ ). The calculated monomer cross sections were used in this work to reliably calibrate the absolute concentrations of monomers in each sample prior to aggregation, which serve to fix the $c_{\text {tot }}$ parameter (Eqn. 9) in the chemometric procedure.

Table 2. Comparison of the ratios of optical-to-physical cross sections $\left(Q_{\text {ext }}\right)$ for light extinction between the monomers and the dimers. The values reported for the $20 \mathrm{~nm}$ LA-AuNS samples are for ionic strength $6.7 \times 10^{-3}$. The extinction cross sections for the monomers were calculated using Mie theory (see text). Calculations for dimers are results from numerical calculations in the discrete-dipole approximation (see text).

\begin{tabular}{|c|c|c|c|c|c|c|}
\hline \multirow{2}{*}{$\begin{array}{l}\text { NS } \\
\text { diam. }\end{array}$} & \multicolumn{2}{|c|}{ monomer } & \multicolumn{4}{|c|}{ dimer (longitudinal) } \\
\hline & $\sigma_{\text {ext }} / \mathrm{nm}^{2}$ & $Q_{\text {ext }}$ & $\begin{array}{c}\sigma_{\text {ext }} / \mathrm{nm}^{2} \\
(\exp .)\end{array}$ & $\begin{array}{c}\sigma_{\mathrm{ext}} / \mathrm{nm}^{2} \\
(\mathrm{DDA})\end{array}$ & $\begin{array}{c}Q_{\text {ext }} \\
(\text { exp. })\end{array}$ & $\begin{array}{c}\mathrm{Q}_{\mathrm{ext}} \\
(\mathrm{DDA})\end{array}$ \\
\hline $20 \mathrm{~nm}$ & $3.5 \times 10^{2}$ & 1.1 & $6.0 \times 10^{2}$ & $6.0 \times 10^{2}$ & 1.3 & 1.3 \\
\hline $50 \mathrm{~nm}$ & $7.3 \times 10^{3}$ & 3.7 & $8.2 \times 10^{3}$ & $11.5 \times 10^{3}$ & 2.6 & 3.7 \\
\hline $80 \mathrm{~nm}$ & $3.4 \times 10^{4}$ & 6.8 & $2.7 \times 10^{4}$ & $3.79 \times 10^{4}$ & 3.3 & 4.8 \\
\hline
\end{tabular}

The relative extinction cross sections $Q$ of the dimers follow a similar trend as those for the monomer. These relative cross sections are one way for representing these experimentally 
determined cross sections in an illustrative manner. Relevant comparisons with theoretical calculations for these experimentally determined cross sections are possible, as long as the convention used for the relative cross section $Q$ is taken into account. Obviously, comparison with theory may also directly involve the absolute cross section.

The experimental absolute extinction cross sections can be compared to results of numerical calculations. As will be detailed below we used an open-source software implementation of the dipole-dipole approximation (DDA) method, DDSCAT ${ }^{48}$ In the calculations we used idealised dimers of perfectly spherical particles of a single precise diameter held at a fixed distance, whereas the experimental spectra are ensemble averages over population distributions, where heterogeneities (in shapes, sizes and interparticle distances) contribute to broadening of the spectral bands. Taking this into consideration, the agreement between the experimental spectra (black solid lines) and the numerical results (blue dotted lines) shown in Figure 9 is good. The extinction cross sections are well reproduced by DDSCAT, taking into account the experimental uncertainties. Also, the positions of the resonance maxima are reliably calculated. An interparticle gap of $1 \mathrm{~nm}$ was used for the calculated spectra in Figure 9. This value was found to give the closest general agreement with experiment after comparing results for calculations using various interparticle gaps (see Supporting Info, Section SI-7.2). This $1 \mathrm{~nm}$ gap is well in line with a gap expected for particles functionalised with lipoic acid.

DDA, a frequency-domain method, was chosen since a robust, and well-tested open-source software implementation, DDSCAT, ${ }^{48}$ exists. The use of DDSCAT for the calculation of plasmonic nanostructures is well documented. ${ }^{49,50,67}$ Other numerical and analytical methods for calculating the interaction of electromagnetic waves with non spherical plasmonic assemblies have been described: frequency-domain methods such as the Boundary Element Method (BEM) $)^{68}$ and Generalised Multiparticle Mie theory ${ }^{64,69,70}$ (GMM, with MESME ${ }^{71}$ as a particular implementation), and time-domain methods such as Finite Difference Time Domain (FDTD). ${ }^{72,73}$

It is essential to compare results of different theoretical methods, ${ }^{74}$ since even for conceptually simple assemblies such as dimers problems with the validity of the approximations used in the models may arise. DDA is a method for which the particle under study is discretized into small cubes, each containing a (discrete) dipole. The calculations become more accurate when the mesh is fine compared to the structure of the particle (i.e. a 
large number of discrete dipoles). Nevertheless, the curvature of the surface cannot be perfectly reproduced whatever the number of dipoles and this is also the case for the structure of the dimer near the gap. As a result, as the particles in the dimers get closer to each other, the accuracy decreases. Moreover, in the case of very small gaps $(\sim 0.5 \mathrm{~nm})$, effects such as electron tunneling come into play and quantum-corrected calculations are needed. ${ }^{75}$ Such effects were not considered here.

A comparison was made between results from DDA (using DDSCAT) and results published by Khlebtsov et al. ${ }^{76}$ obtained using GMM. This comparison was done for dimers of $30 \mathrm{~nm}$ particles, a choice imposed by the literature data. Details of the comparison are provided in the Supporting Info (Section SI-7.1). There is good agreement between the results for GMM and DDA with respect to the absolute cross sections, with slight differences in the positions of the resonance maxima, $\Delta \lambda \approx 15 \mathrm{~nm}$. The results are coherent between different numerical methods, and between the numerical results and experimental data.

\subsection{Scattering cross sections of gold nanosphere dimers in water}

The time-evolution of the plasmon resonances of dimerising nanosphere suspension is also evident in resonant light scattering (RLS) spectra. This is illustrated in Figure 10 for the case of the dimerisation of $80 \mathrm{~nm}$ diameter LA-coated gold nanospheres. RLS spectroscopy requires low optical densities (< 0.05 throughout the spectrum), and the corresponding low particle concentrations lead to slower aggregation. Hence, the aggregation conditions were adapted by strongly increasing ionic strength using $\mathrm{NaCl}$, while staying at $\mathrm{pH}$ 5.0. The timeresolved RLS spectral data are readily analysed using the same chemometric procedure as used for the extinction spectra. This was done for gold nanoparticles of $20 \mathrm{~nm}, 50 \mathrm{~nm}$ and 80 nm diameter (Fig. 10, and Figs. S8 and S9, Supporting Info). 

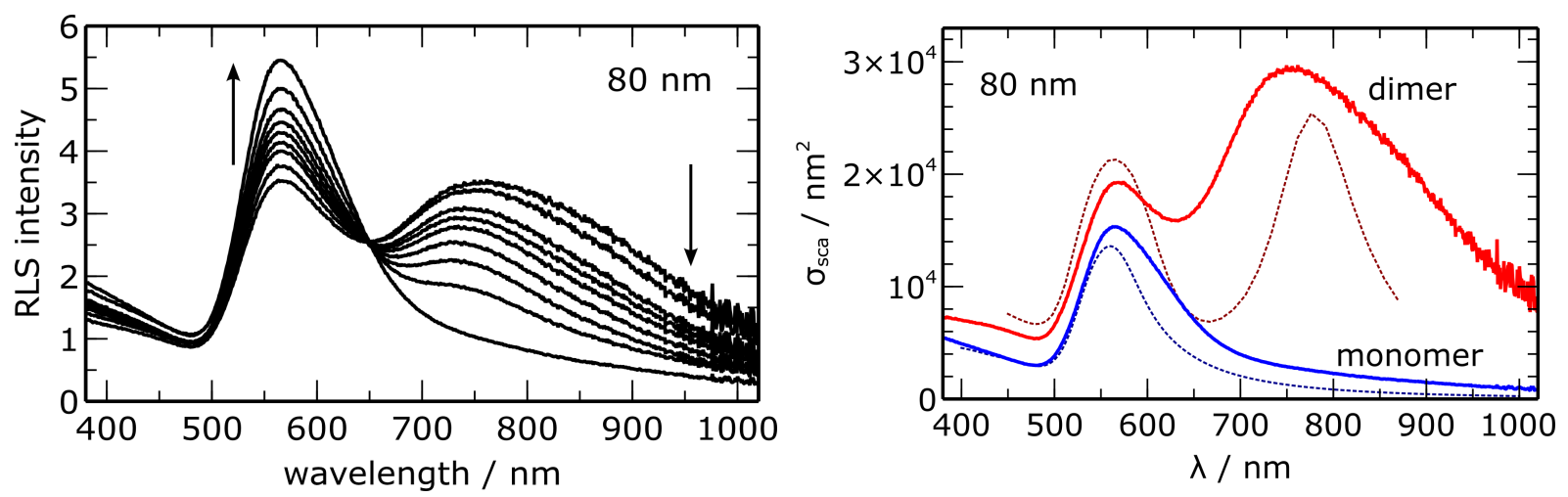

Figure 10. Left: Time-evolution of the resonant light scattering spectrum of dimerising $80 \mathrm{~nm}$ LA-AuNS. Aggregation was induced by changing the $\mathrm{pH}$ of the suspension to 5.0 and the ionic strength to 0.05 by adding $1 \mathrm{~mL}$ of concentrated saline acetate buffer to $2 \mathrm{~mL}$ of $2.2 \times 10^{-13} \mathrm{M}$ AuLA $(80 \mathrm{~nm})$ suspension in $1 \mathrm{mM} \mathrm{NaOH}$. Right: decomposition into individual components, i.e. experimental spectra with absolute scattering cross sections for monomers and dimers (solid lines). The dotted lines are theoretical absolute cross sections for ideal $80 \mathrm{~nm}$ diameter gold spheres in water obtained using analytical Mie theory (monomer) and numerical DDA calculation (dimer interparticle gap $1 \mathrm{~nm}$ ).

Compared to the light extinction cross sections, the relative cross-sections ( $\mathrm{Q}_{\text {sca }}$, Table 3) for resonant light scattering by monomers and dimers of spherical gold particles are much more strongly dependent on the particle diameters. Moreover, the contrast in light scattering cross section between monomer and dimer is higher, in particular for the smaller particles, and at the wavelengths near the maximum of the longitudinal plasmon of the dimer. 
Table 3. Comparison of the ratios of optical-to-physical cross sections for light extinction between the monomers and the dimers. The $\mathrm{Q}_{\text {sca }}(\mathrm{Mie})$ for monomers were calculated using Mie theory (see Text). $\mathrm{Q}_{\text {sca }}(\mathrm{DDA})$ for dimers were calculated using DDSCAT 7.3 (see Text) using an interparticle gap of $1 \mathrm{~nm}$. The values reported for the $20 \mathrm{~nm}$ LA-AuNS samples are for ionic strength $6.7 \times 10^{-3}$.

\begin{tabular}{lccccccc}
\hline NS diameter & \multicolumn{3}{c}{ monomer (max.) } & \multicolumn{4}{c}{ dimer (longitudinal, max.) } \\
& $\sigma_{\text {sca }} / \mathrm{nm}^{2}$ & $\begin{array}{c}\mathrm{Q}_{\text {sca }} \\
(\text { exp. })\end{array}$ & $\begin{array}{c}\mathrm{Q}_{\text {sca }} \\
(\mathrm{Mie})\end{array}$ & $\begin{array}{c}\sigma_{\text {sca }} / \mathrm{nm}^{2} \\
(\text { exp.) }\end{array}$ & $\begin{array}{c}\sigma_{\text {sca }} / \mathrm{nm}^{2} \\
(\mathrm{DDA})\end{array}$ & $\begin{array}{c}\mathrm{Q}_{\text {sca }} \\
(\mathrm{exp} .)\end{array}$ & $\begin{array}{c}\mathrm{Q}_{\text {sca }} \\
(\mathrm{DDA})\end{array}$ \\
\hline $20 \mathrm{~nm}$ & 4 & 0.01 & 0.01 & 33 & 14 & 0.08 & 0.03 \\
$50 \mathrm{~nm}$ & $1.0 \times 10^{3}$ & 0.51 & 0.47 & $4.1 \times 10^{3}$ & $4.1 \times 10^{3}$ & 1.2 & 1.2 \\
$80 \mathrm{~nm}$ & $1.5 \times 10^{4}$ & 3.0 & 2.7 & $2.9 \times 10^{4}$ & $2.4 \times 10^{4}$ & 3.6 & 3.0 \\
\hline
\end{tabular}

Good agreement exists in general between the experimental and the calculated cross sections, which is not only illustrated by Table 3, but also by the theoretical scattering spectra included with the experimental spectra in Figure 10 (right). The numerical calculations consider idealised dimers consisting of spherical gold nanoparticles of exactly the same precise diameters with constant interparticle gap in a pure water-like dielectric environment. Heterogeneities in size and shape were not considered at this stage. These heterogeneities are most likely the source of the broadening of the observed spectral resonances. Also, the effect of the ligand shell was not modeled. Since we work with small molecule ligands, only a small effect of the ligands on the plasmon resonances of the particles and dimers is expected. This is evidenced by the agreement in absolute cross sections and spectral positions of the plasmon resonance between the experimental data and the numerical modeling.

This agreement between theory and the values deduced from chemometric analysis of the experimental data, both for light extinction and scattering, further strengthens our quantitative spectroscopic analysis of these gold nanoparticle dimers, and validates our experimental approach to the point that it now becomes feasible to study more complex colloidal plasmonic objects, and to consider the more subtle effects of the ligand shell on the optical properties of the assemblies.

From the extinction and scattering cross sections we can furthermore calculate the quantum efficiencies of light scattering by the dimers at the plasmon maximum $\eta_{\text {sca }}=\sigma_{\text {sca }} / \sigma_{\text {ext }}$, i.e. the fraction of interacting photons that is scattered (Table 4). The remainder, $1-\eta_{\text {sca }}$, is absorbed 
by the dimer and is practically completely converted into heat (except for a tiny fraction of the energy that may escape in the form of photoluminescence ${ }^{39,77,78}$ ). This is relevant for photothermal $^{79,80}$ applications, as this provides a quantitative measure of how much of the light interacting with the nanoparticles is finally transformed into heat.

Table 4. Scattering efficiencies $\eta_{\text {sca }}=\sigma_{\text {sca }} / \sigma_{\text {ext }}$ at the wavelengths of the maxima corresponding to the transverse and longitudinal resonances, respectively. The values reported for the $20 \mathrm{~nm}$ LA-AuNS samples are for ionic strength $6.7 \times 10^{-3}$.

\begin{tabular}{lcccccc}
\hline & \multicolumn{3}{c}{ transverse resonance } & \multicolumn{3}{c}{ longitudinal resonance } \\
$\begin{array}{l}\text { NS } \\
\text { diam. } \\
(\mathrm{nm})\end{array}$ & $\lambda_{\max }(\mathrm{nm})$ & $\sigma_{\text {sca }}\left(\mathrm{nm}^{2}\right)$ & $\eta_{\text {sca }}$ & $\begin{array}{c}\lambda_{\max } \\
(\mathrm{nm})\end{array}$ & $\sigma_{\text {sca }}\left(\mathrm{nm}^{2}\right)$ & $\eta_{\text {sca }}$ \\
\hline $20 \mathrm{~nm}$ & $550(\mathrm{sh})$ & 16 & 0.02 & 690 & 33 & 0.05 \\
$50 \mathrm{~nm}$ & 550 & $2.5 \times 10^{3}$ & 0.21 & 681 & $4.1 \times 10^{3}$ & 0.50 \\
$80 \mathrm{~nm}$ & 568 & $1.9 \times 10^{4}$ & 0.43 & 752 & $2.9 \times 10^{4}$ & 1.0
\end{tabular}

Interesting is the difference in scattering efficiencies between the shorter-wavelength transverse plasmon band and the long-wavelength longitudinal plasmon band. Photothermal heating in these dimers can be expected to be more efficient for shorter wavelengths, whereas resonant light scattering is more efficient at longer wavelengths. This shows that a combined quantitative study of extinction and scattering spectra will help in identifying the degree of tunability between different light-induced effects (here, photothermal vs. light scattering) within a particular colloidal plasmonic structure. Such information is of particular relevance for the development of plasmonic materials for combined cell imaging and photothermal therapy using near-infrared light. ${ }^{81}$

\section{Conclusion}

The spectrometric analysis of suspensions of slowly aggregating colloids provides a convenient method for obtaining quantitative data on the spectroscopic behaviour of small assemblies of plasmonic nanoparticles. This method may be useful for identifying combinations of nanoparticles with desired optical properties prior to more elaborate synthesis and purification of assemblies (e.g. using DNA as a scaffold ${ }^{29}$ ). In the present work, the chemometric analysis (which with present-day computational power is readily and rapidly carried out) was applied to the study of plasmonic dimers in an aqueous system 
containing lipoic acid as a proton-sensitive ligand. This colloidal system provides an interesting plasmonic playground, enabling dimer formation for particles up to (at least) 80 $\mathrm{nm}$. It was found that ionic strength and stabilising ligand concentration influences to some extent the optical spectra of the dimers, which is of relevance for the design of plasmonic biosensors based on dimers.

The identification of gold nanoparticle dimers in this work relies on four independent observations. First, the occurrence of an isosbestic point in the optical spectra indicates that the aggregation reaction takes place at constant stoichiometry. Since it would be very unlikely for the initially monomeric gold nanoparticles to directly assemble exclusively into trimers or higher aggregates, the constant stoichiometry should be the monomer-to-dimer conversion. We emphasize that this optical spectroscopic identification relies on the chemometric analysis of the evolution of a series of spectra of the same sample evolving over time, not exclusively on a single spectrum of the final aggregated state. Singular-value decomposition of the spectrum $v s$ time matrix for the time ranges of initial aggregation always indicates only two principal components.

Second, the diffusion of the newly formed species coefficient as measured by light scattering correlation spectroscopy correspond to that expected for a dimer. In addition, the light scattering correlation of the dimer samples contains a rotational component compatible with a dimer (and incompatible with a monomer, which does not have scattering fluctuations due rotation).

Third, mass spectrometry shows that the aggregating samples contain mainly objects that have a mass that is double the mass of a single particle. This is the first time that plasmonic nanoassemblies have been characterised directly by mass spectrometry.

Finally, the close agreement between experimental absolute absorption and scattering cross section spectra and those calculated numerically for idealised dimers corroborates the identification of the gold nanoparticle dimers.

The method presented here allows for extraction of absolute cross sections for light extinction and scattering from the spectra of simple mixtures of self-assembling plasmonic objects. It may likely be extended to more complicated systems, provided that the spectra of the individual components are sufficiently distinct, and the chemistry of the system is welldefined. The absolute values for the optical cross sections obtained are of use for designing 
and evaluating detection schemes based on plasmonic shifts, ${ }^{15,23}$ and in other applications of colloidal plasmonics.

\section{Acknowledgements}

Financial support from the Agence Nationale de Recherche through grants ANR-2010-JCJC1005-1 (COMONSENS), ANR-08-BLAN-0110-01 (LUMinNANOH), ANR-11-PDOC-032-01 (PONAME), and ANR-10-LABX-0035 (LabEx NanoSaclay) is gratefully acknowledged. MS and $\mathrm{AD}$ received financial support by the Région Ile-de-France in the framework of C'Nano IdF.

\section{Appendix: definition of symbols}

$\mathrm{A}_{\mathrm{H}} \quad$ Hamaker constant. $\left[2.5 \times 10^{-19} \mathrm{~J}_{\text {for }}\right.$ gold $\left.^{8}\right]$

a particle radius $[\mathrm{m}]$

$b^{\varnothing} \quad 1 \mathrm{~mol} \mathrm{~kg}^{-1}$

$c \quad$ particle concentration $\left[\mathrm{mol} \mathrm{L}^{-1}\right]$

$d \quad$ particle diameter $\left(d=2 a \times 10^{9}\right)[\mathrm{nm}]$

$\varepsilon_{0} \varepsilon_{\mathrm{r}} \quad$ medium permittivity [78 $\mathrm{x} 8.854 \cdot 10^{-12} \mathrm{~F} \mathrm{~m}^{-1}$, water]

$\varepsilon_{\max } \quad$ maximum extinction coefficient $\left[\mathrm{M}^{-1} \mathrm{~cm}^{-1}\right]$

g gravitational acceleration $\left[9.81 \mathrm{~m} \mathrm{~s}^{-2}\right]$

I $\quad$ ionic strength

$I_{\mathrm{LS}} \quad$ instrument-corrected light-scattering intensity

$k_{\mathrm{B}} \quad$ Boltzmann constant $\left[1.381 \times 10^{-23} \mathrm{~J} \mathrm{~K}^{-1}\right]$

$K_{\mathrm{LJ}} \quad$ strength of the steric repulsion due to the molecular layer [J]

$\lambda \quad$ wavelength $[\mathrm{nm}]$

$N_{\mathrm{A}} \quad$ Avogadro constant $\left[6.022 \times 10^{23} \mathrm{~mol}^{-1}\right]$

$N_{\text {sites }}$ number of thiol binding sites per particle

$R \quad$ center-to-center distance between two particles, $R=s+2 a[\mathrm{~m}]$

$r_{\mathrm{D}} \quad$ Debye length [m] 


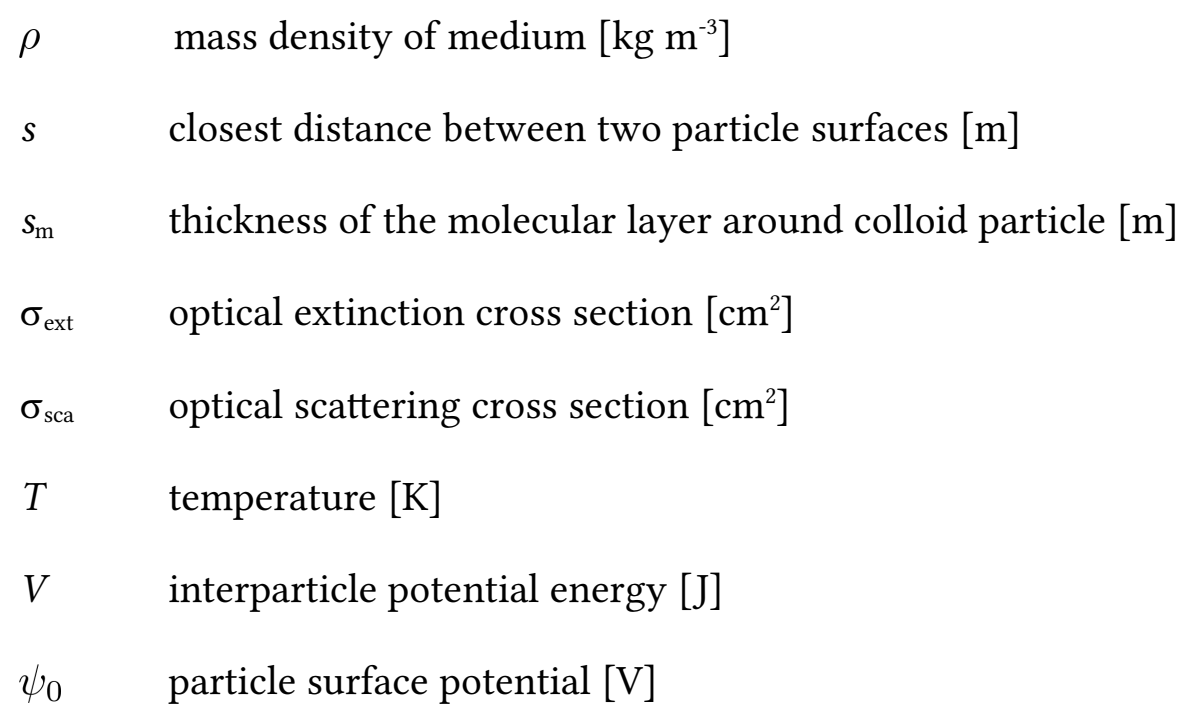

\section{References}

1 R. Fernandes, M. Li, E. Dujardin, S. Mann and A. G. Kanaras, Chem. Commun., 2010, 46, $7602-4$.

2 S. Mühlig, A. Cunningham, J. Dintinger, T. Scharf, T. Bürgi, F. Lederer and C. Rockstuhl, Nanophotonics, 2013, 2, 211-240.

3 R. Esteban, R. W. Taylor, J. J. Baumberg and J. Aizpurua, Langmuir, 2012, 28, 8881-90.

4 S. J. Barrow, A. M. Funston, X. Wei and P. Mulvaney, Nano Today, 2013, 8, 138-167.

5 J. M. Romo-Herrera, R. A. Alvarez-Puebla and L. M. Liz-Marzán, Nanoscale, 2011, 3, 130415 .

6 F. Kretschmer, M. Fruhnert, R. Geiss, U. Mansfeld, C. Höppener, S. Hoeppener, C. Rockstuhl, T. Pertsch and U. S. Schubert, f. Mater. Chem. C, 2014, 2, 6415-6422.

7 P. Dimon, S. K. Sinha, D. A. Weitz, C. R. Safinya, G. S. Smith, W. A. Varady and H. M. Lindsay, Phys. Rev. Lett., 1986, 57, 595-598.

8 T. Kim, C.-H. Lee, S.-W. Joo and K. Lee, J. Colloid Interface Sci., 2008, 318, 238-43.

9 J. van Rijssel, B. H. Erné, J. D. Meeldijk, M. Casavola, D. Vanmaekelbergh, A. Meijerink and A. P. Philipse, Phys. Chem. Chem. Phys., 2011, 13, 12770-4.

10 S. M. Heard, F. Grieser, C. G. Barraclough and J. V Sanders, f. Colloid Interface Sci., 1983, 93, 545-555.

11 J. A. Molina-Bolívar and F. Galisteo-González, f. Macromol. Sci. Part C Polym. Rev., 2005, 45, 59-98.

12 S. Gupta, P. K. Kilpatrick, E. Melvin and O. D. Velev, Lab Chip, 2012, 12, 4279-86.

13 N. T. K. Thanh and Z. Rosenzweig, Anal. Chem., 2002, 74, 1624-1628.

14 J. J. Storhoff, A. D. Lucas, V. Garimella, Y. P. Bao and U. R. Müller, Nat. Biotechnol., 2004, 
$22,883-887$.

15 L. Lermusiaux, V. Maillard and S. Bidault, ACS Nano, 2015, 9, 978-990.

16 P. Englebienne, Analyst, 1998, 123, 1599-603.

17 J. R. Kalluri, T. Arbneshi, S. A. Khan, A. Neely, P. Candice, B. Varisli, M. Washington, S. McAfee, B. Robinson, S. Banerjee, A. K. Singh, D. Senapati and P. C. Ray, Angew. Chemie Int. Ed., 2009, 48, 9668-71.

18 K. Ai, Y. Liu and L. Lu, f. Am. Chem. Soc., 2009, 131, 9496-7.

19 C. Han and H. Li, Analyst, 2010, 135, 583-8.

20 D. Su, X. Yang, Q. Xia, F. Chai, C. Wang and F. Qu, RSC Adv., 2013, 3, 24618.

21 S. Basu, S. K. Ghosh, S. Kundu, S. Panigrahi, S. Praharaj, S. Pande, S. Jana and T. Pal, f. Colloid Interface Sci., 2007, 313, 724-34.

22 J. R. G. Navarro and M. H. V Werts, Analyst, 2013, 138, 583-592.

23 K. L. Göeken, V. Subramaniam and R. Gill, Phys. Chem. Chem. Phys., 2015, 17, 422-427.

24 M. Horisberger and J. Rosset, f. Histochem. Cytochem., 1977, 25, 295-305.

25 N. Nerambourg, R. Praho, M. H. V. Werts, D. Thomas and M. Blanchard-Desce, Int. $\mathcal{F}$. Nanotechnol., 2008, 5, 722.

26 L. Lermusiaux, A. Sereda, B. Portier, E. Larquet and S. Bidault, ACS Nano, 2012, 6, 109928.

27 S. Bidault and A. Polman, Int. F. Opt., 2012, 2012, 1-5.

28 G. Chen, Y. Wang, L. H. Tan, M. Yang, L. S. Tan, Y. Chen and H. Chen, F. Am. Chem. Soc., 2009, 131, 4218-9.

29 A. Heuer-Jungemann, R. Kirkwood, A. H. El-Sagheer, T. Brown and A. G. Kanaras, Nanoscale, 2013, 5, 7209-12.

30 A. Lombardi, M. Loumaigne, A. Crut, P. Maioli, N. Del Fatti, F. Vallée, M. Spuch-Calvar, J. Burgin, J. Majimel and M. Tréguer-Delapierre, Langmuir, 2012, 28, 9027-33.

31 S.-Y. Lin, Y.-T. Tsai, C.-C. Chen, C.-M. Lin and C. Chen, f. Phys. Chem. B, 2004, 108, 21342139.

32 A. Lombardi, M. P. Grzelczak, A. Crut, P. Maioli, I. Pastoriza-Santos, L. M. Liz-Marzán, N. Del Fatti and F. Vallée, ACS Nano, 2013, 7, 2522-2531.

33 T. Doussineau, C. Y. Bao, R. Antoine, P. Dugourd, W. Zhang, F. D’Agosto and B. Charleux, ACS Macro Lett., 2012, 1, 414-417.

34 T. Doussineau, C. Mathevon, L. Altamura, C. Vendrely, P. Dugourd, V. Forge and R. Antoine, Angew. Chemie Int. Ed., 2016, 55, 2340-2344.

35 T. Doussineau, A. Désert, O. Lambert, J.-C. Taveau, M. Lansalot, P. Dugourd, E. Bourgeat- 
Lami, S. Ravaine, E. Duguet and R. Antoine, J. Phys. Chem. C, 2015, 119, 10844-10849.

36 I. Chaduc, J. Parvole, T. Doussineau, R. Antoine, A. Désert, P.-Y. Dugas, S. Ravaine, E. Duguet, E. Bourgeat-Lami and M. Lansalot, Polymer, 2015, 70, 118-126.

37 G. Frens, Nat. Phys. Sci., 1973, 241, 20-22.

38 J. Turkevich, P. C. Stevenson and J. Hillier, Discuss. Faraday. Soc, 1951, 11, 55-75.

39 M. Loumaigne, J. R. G. Navarro, S. Parola, M. H. V. Werts and A. Débarre, Nanoscale, 2015, 7, 9013-9024.

40 G. Deželić and J. P. Kratohvil, Kolloid-Zeitschrift, 1960, 173, 38-48.

41 M. H. V. Werts, "Luminescent Lanthanide Complexes: Visible Light Sensitised Red and Near-infrared Luminescence", Ph. D. thesis, University of Amsterdam, 2000.

42 M. Kubista, Chemom. Intell. Lab. Syst., 1990, 7, 273-279.

43 M. Loumaigne, P. Vasanthakumar, A. Richard and A. Débarre, Phys. Chem. Chem. Phys., 2011, 13, 11597-11605.

44 T. Doussineau, C. Yu Bao, C. Clavier, X. Dagany, M. Kerleroux, R. Antoine and P. Dugourd, Rev. Sci. Instrum., 2011, 82.

45 C. F. Bohren and D. R. Huffman, Absorption and scattering of light by small particles, Wiley Interscience, New York, 1983.

46 P. B. Johnson and R. W. Christy, Phys. Rev. B, 1972, 6, 4370-4379.

47 W. Haiss, N. T. K. Thanh, J. Aveyard and D. G. Fernig, Anal. Chem., 2007, 79, 4215-21.

48 B. T. Draine and P. J. Flatau, f. Opt. Soc. Am. A, 1994, 11, 1491.

49 M. A. Yurkin, D. De Kanter and A. G. Hoekstra, F. Nanophotonics, 2010, 4, 041585.

50 M. A. Yurkin, M. Min and A. G. Hoekstra, Phys. Rev. E, 2010, 82, 036703.

51 IUPAC, IUPAC Gold book. Compendium of Chemical Terminology, 2012.

52 R. J. Hunter, Foundations of colloid science, Oxford University Press, Oxford, UK, 2nd edn., 2001.

53 S. Dominguez-Medina, J. Blankenburg, J. Olson, C. F. Landes and S. Link, ACS Sustain. Chem. Eng., 2013, 1, 833-842.

54 M. M. Tirado, C. L. Martínez and J. G. de la Torre, f. Chem. Phys., 1984, 81, 2047.

55 J. Garcia de la Torre, G. del Rio Echenique and A. Ortega, f. Phys. Chem. B, 2007, 111, 955-961.

56 E. E. Pierson, D. Z. Keifer, L. Selzer, L. S. Lee, N. C. Contino, J. C. Y. Wang, A. Zlotnick and M. F. Jarrold, F. Am. Chem. Soc., 2014, 136, 3536-3541.

57 P. W. Atkins and J. De Paula, Physical Chemistry, 9th Edition, W. H. Freeman, 2009. 
58 H. C. Hamaker, Physica, 1937, 4, 1058-1072.

59 B. W. Ninham, Adv. Colloid Interface Sci., 1999, 83, 1-17.

60 K. J. M. Bishop, C. E. Wilmer, S. Soh and B. A. Grzybowski, Small, 2009, 5, 1600-1630.

61 A. R. Smith, S. V Shenvi, M. Widlansky, J. H. Suh and T. M. Hagen, Curr. Med. Chem., 2004, 11, 1135-1146.

62 M. Rooth and A. M. Shaw, Phys. Chem. Chem. Phys., 2006, 8, 4741-4743.

63 P. K. Jain and M. A. El-Sayed, F. Phys. Chem. C, 2008, 112, 4954-4960.

64 B. Khlebtsov, A. Melnikov, V. Zharov and N. Khlebtsov, Nanotechnology, 2006, 17, $1437-$ 1445.

65 X. Liu, M. Atwater, J. Wang and Q. Huo, Colloids Surf. B. Biointerfaces, 2007, 58, 3-7.

66 N. G. Khlebtsov, Anal. Chem., 2008, 80, 6620-6625.

67 C. Ungureanu, R. G. Rayavarapu, S. Manohar and T. G. van Leeuwen, F. Appl. Phys., 2009, 105, 102032.

68 V. Myroshnychenko, E. Carbó-Argibay, I. Pastoriza-Santos, J. Pérez-Juste, L. M. LizMarzán and F. J. García de Abajo, Adv. Mater., 2008, 20, 4288-4293.

69 Y. Xu and N. G. Khlebtsov, F. Quant. Spectrosc. Radiat. Transf., 2003, 79-80, 1121-1137.

70 F. J. García de Abajo, Phys. Rev. Lett., 1999, 82, 2776-2779.

71 F. J. García de Abajo, Phys. Rev. B, 1999, 60, 6086-6102.

72 A. Dhawan, S. J. Norton, M. D. Gerhold and T. Vo-Dinh, Opt. Express, 2009, 17, 9688-9703.

73 C. Oubre and P. Nordlander, F. Phys. Chem. B, 2005, 109, 10042-10051.

74 V. Myroshnychenko, J. Rodríguez-Fernández, I. Pastoriza-Santos, A. M. Funston, C. Novo, P. Mulvaney, L. M. Liz-Marzán and F. J. García de Abajo, Chem. Soc. Rev., 2008, 37, 1792.

75 R. Esteban, A. G. Borisov, P. Nordlander and J. Aizpurua, Nat. Commun., 2012, 3, 825.

76 B. Khlebtsov, A. Melnikov, V. Zharov and N. Khlebtsov, Nanotechnology, 2006, 17, 14371445.

77 J. P. Wilcoxon, J. E. Martin, F. Parsapour, B. Wiedenman and D. F. Kelley, f. Chem. Phys., 1998, 108, 9137-9143.

78 M. Yorulmaz, S. Khatua, P. Zijlstra, A. Gaiduk and M. Orrit, Nano Lett., 2012, 12, 4385-91.

79 X. Huang, P. K. Jain, I. H. El-Sayed and M. A. El-Sayed, Lasers Med. Sci., 2008, 23, 217-228.

80 A. M. Alkilany, L. B. Thompson, S. P. Boulos, P. N. Sisco and C. J. Murphy, Adv. Drug Deliv. Rev., 2012, 64, 190-199.

81 X. Huang, I. H. El-Sayed, W. Qian and M. A. El-Sayed, F. Am. Chem. Soc., 2006, 128, 21152120. 


\section{- Supporting Information -}

\section{Optical extinction and scattering cross sections of plasmonic nanoparticle dimers in aqueous suspension}

Matthieu Loumaigne[1], Clyde Midelet[1], Tristan Doussineau[2], Philippe Dugourd[2],

Rodolphe Antoine[2], Meriem Stamboul[3], Anne Débarre[3][4] and Martinus H. V. Werts*[1]

[1] École normale supérieure de Rennes, CNRS, SATIE (UMR 8029), Campus de Ker Lann, F35170 Bruz, France

[2] Université de Lyon 1, CNRS, Institut Lumière Matière (UMR 5306), F-69622 Villeurbanne Cedex, France

[3] Laboratoire Aimé Cotton, CNRS, Univ. Paris-Sud, ENS Cachan, Université Paris-Saclay, 91405 Orsay Cedex, France

[4] École normale supérieure de Cachan, CNRS, PPSM (UMR 8531), F-94235 Cachan, France.

*) e-mail: martinus.werts@ens-rennes.fr 


\section{SI-1 Singular-value decomposition of an experimental spectrotemporal extinction matrix}
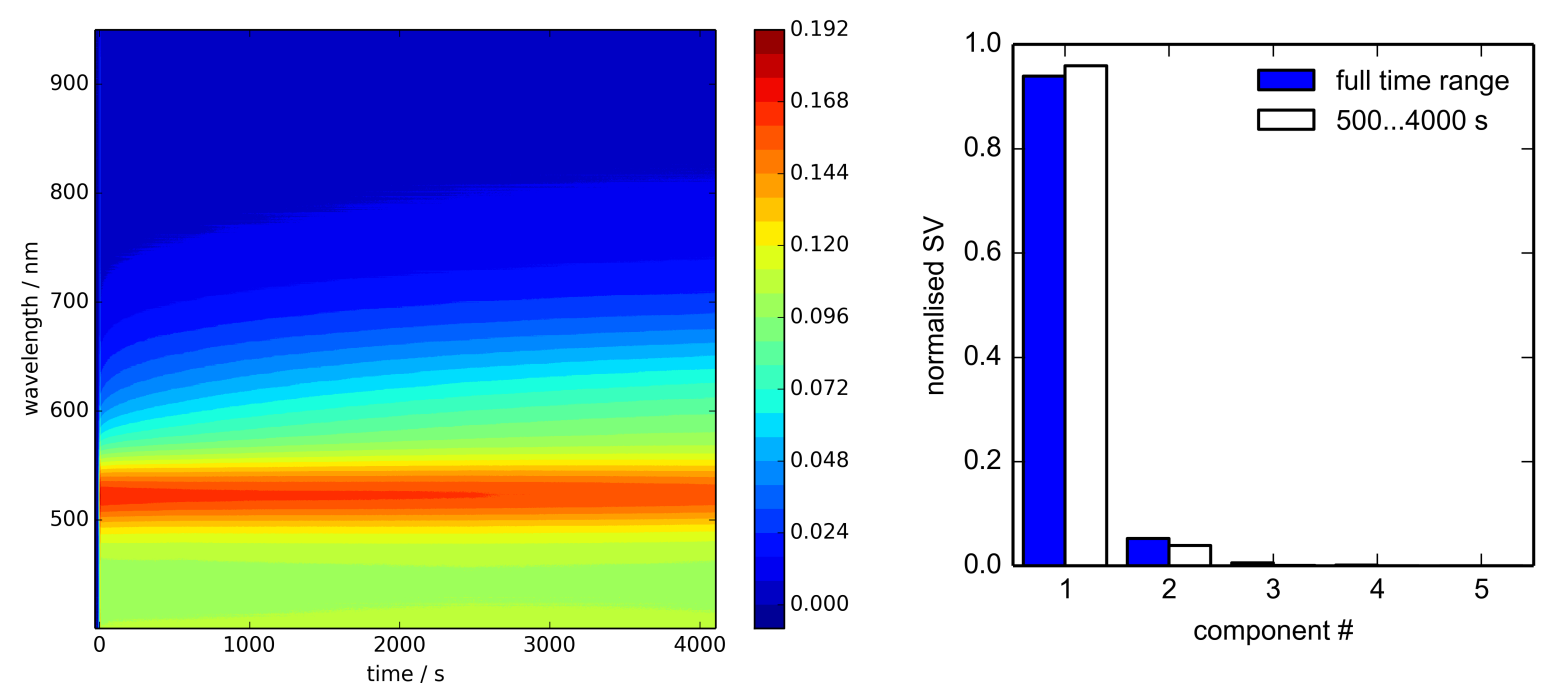

Figure S1. SVD analysis of the experimental data set from Figure 1 of the main text. Left: Time-evolution of the extinction spectra of $20 \mathrm{~nm}$ LA-AuNS in acetate buffer at $\mathrm{pH}$ 5.1, ionic strength $I=4.8 \times 10^{-3}$. Right: Normalised singular values (SV) obtained from singular-value decomposition: blue: SVD over the entire data range, showing a very weak 3rd component; white: SVD over a selected time range $(500 \ldots 4000$ s) showing only 2 principal components. 


\section{SI-2 Control of the aggregation kinetics by $\mathrm{pH}$ and ionic strength}

\section{SI-2.1 Effect of $\mathrm{pH}$ on the aggregation kinetics of LA-AuNS, $20 \mathrm{~nm}$ diameter}

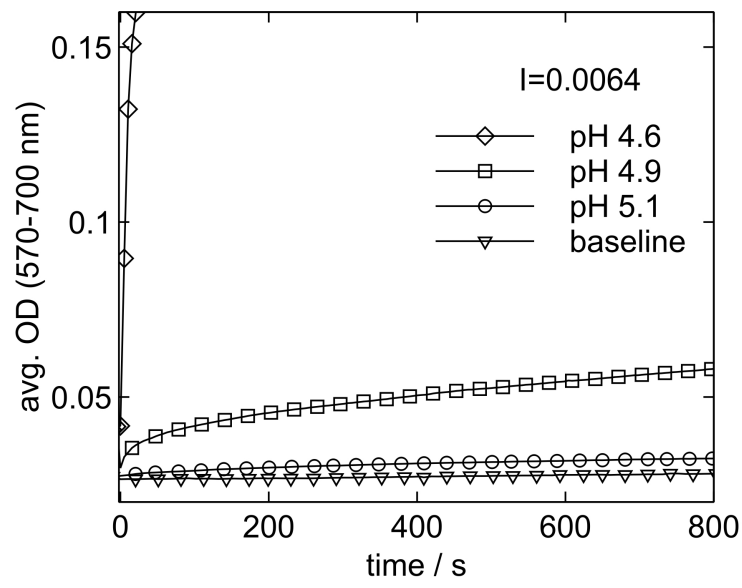

Figure S2. Effect of the $\mathrm{pH}$ on the kinetics of the aggregation, at a constant ionic strength, $I=6.4 \times 10^{-3}$. The reaction was monitored by means of the optical density in the region $570 \ldots 700 \mathrm{~nm}$ corresponding to extinction by gold NS aggregates. A $t=0$, a concentrated stock of buffer was added (1:2 v/v) to a AuLA suspension in $1 \mathrm{mM} \mathrm{NaOH}$. The baseline trace was recorded by adding pure water $(1: 2 \mathrm{v} / \mathrm{v})$ to the basic AuLA suspension.

SI-2.2 Effect of ionic strength on the aggregation kinetics of LA-AuNS, $20 \mathrm{~nm}$ diameter

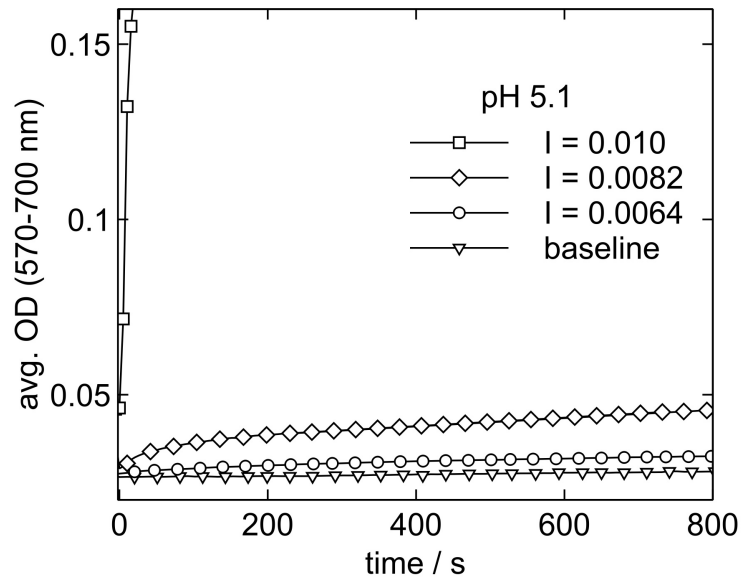

Figure S3. Effect of the ionic strength on the kinetics of the aggregation, at constant $\mathrm{pH}$ 5.1. The ionic strength was changed by changing the buffer concentration. The experimental procedure was identical to Fig. $\mathbf{S 3}$. 


\section{SI-3 Extinction spectroscopy}

\section{SI-3.1 Comparison of extracted LA-AuNS-20nm dimer spectrum with literature}

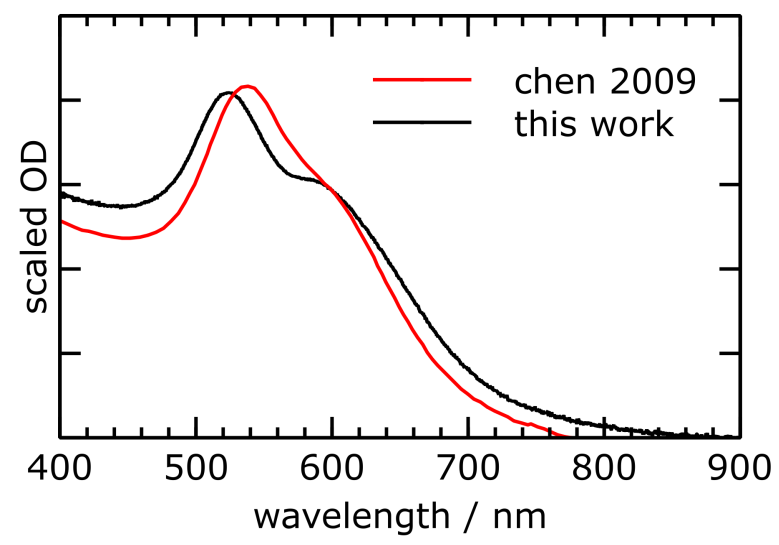

Figure S4. Comparison of extracted dimer extinction spectrum with literature. Red: extinction spectrum of a purified sample of $15 \mathrm{~nm}$ gold nanosphere dimers reported in literature. ${ }^{51}$ Black: Scaled extinction spectrum for dimers of $20 \mathrm{~nm}$ LA-coated gold nanospheres. The literature spectrum was digitised from the published article using the Engauge software package (http://digitizer.sourceforge.net/). The literature spectrum consists only of relative values (i.e. it only represents the spectral shape and does not give absolute cross section values).

\section{SI-3.2 Time-evolution of the extinction spectrum LA-AuNS in presence of excess} LA

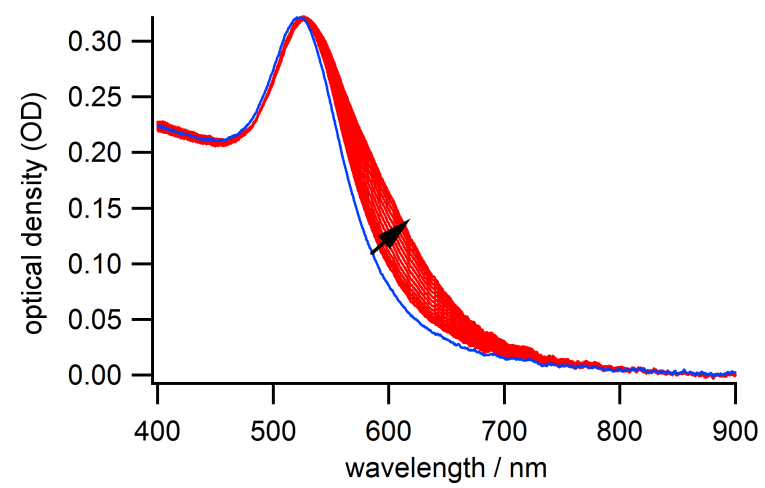

Figure S5. Time-evolution of the extinction spectrum of $20 \mathrm{~nm}$ LA-AuNS in presence of an additional $1 \mathrm{mM}$ of $\mathrm{LA}$ at $\mathrm{pH} 4$. In blue dotted line the initial spectrum of the LAAuNS. The dimer spectrum extracted from these data is in Figure 8 of the main text. 
SI-3.3 Extinction spectra of the formation of dimers of $50 \mathrm{~nm}$ godld nanospheres

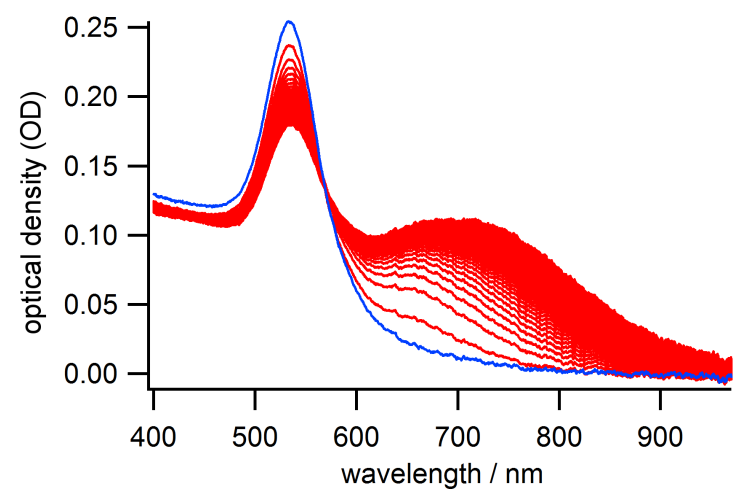

Figure S6. Evolution over time of the extinction spectra of $50 \mathrm{~nm}$ LA-AuNS at $\mathrm{pH} 3.6$ $\left(I=1.1 \times 10^{-3}\right)$

SI-3.4 Extinction spectra of the formation of dimers of $80 \mathrm{~nm}$ gold nanospheres

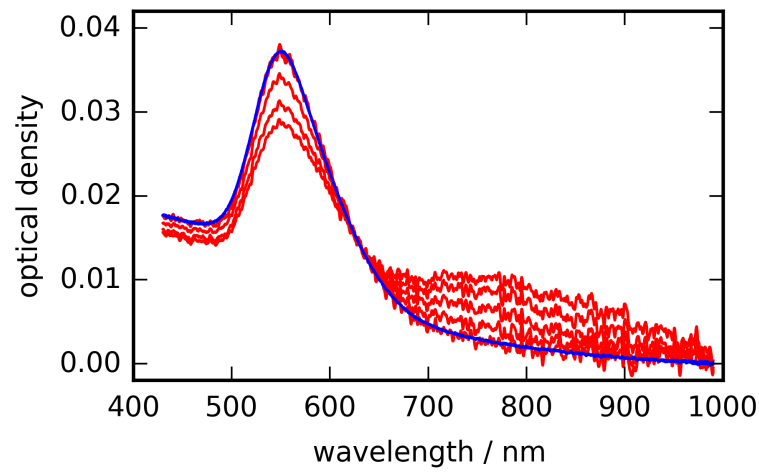

Figure S7. Time-evolution of the extinction spectrum for the slow dimerisation of 80 nm LA-AuNS at $\mathrm{pH} 5.0, I=2.7 \times 10^{-2}$. 


\section{SI-4 Resonant light scattering spectroscopy}

\section{SI-4.1 Resonant light scattering from dimerising $20 \mathrm{~nm}$ gold nanospheres}
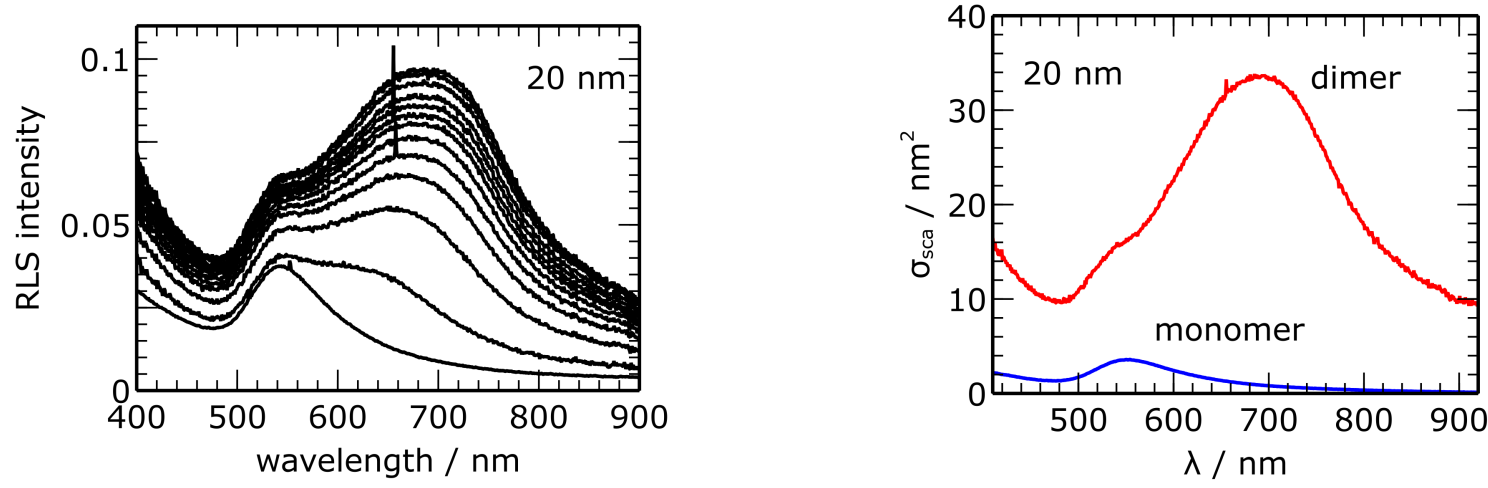

Figure S8. Right: Absolute RLS cross sections for monomers (blue) and dimers (red) of $20 \mathrm{~nm}$ AuLA gold nanospheres, extracted from the spectral evolution data set for nanospheres slowly aggregating in $10 \mathrm{mM}$ acetate buffer $\left(\mathrm{pH} 5.1, I=6.7 \times 10^{-3}\right)$.

\section{SI-4.2 Resonant light scattering from dimerising $50 \mathrm{~nm}$ gold nanospheres}
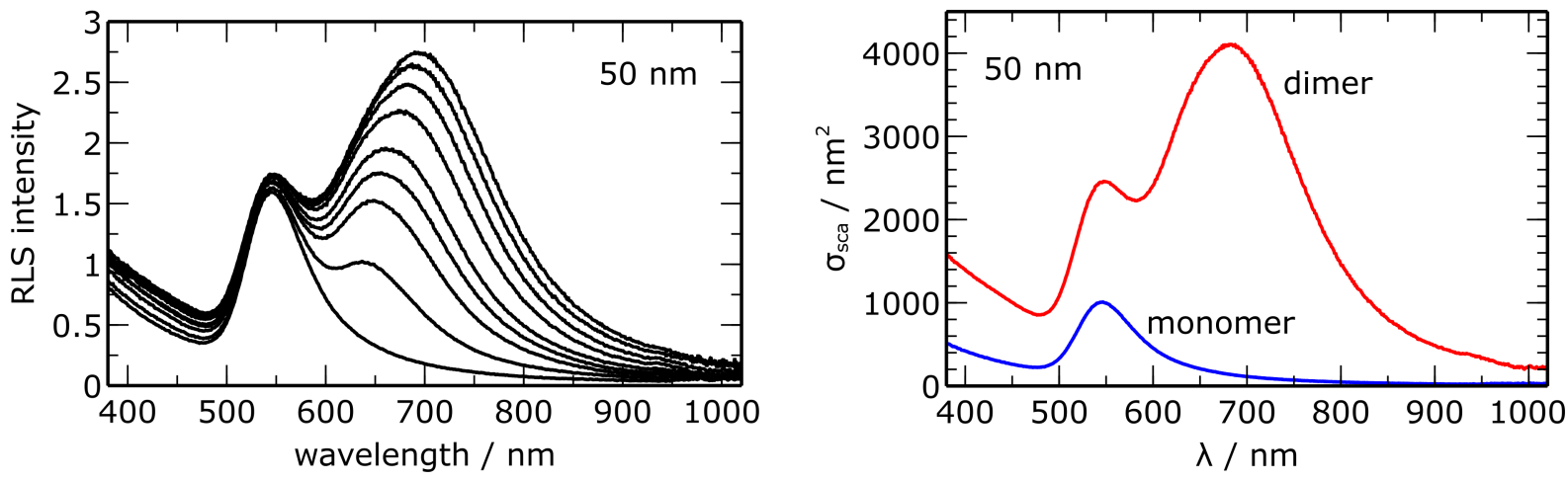

Figure S9. Left: Time-evolution of the resonant light scattering spectrum of dimerising $50 \mathrm{~nm}$ LA-AuNS. Right: decomposition into individual components, spectra with absolute scattering cross sections for monomers and dimers. Aggregation was induced by changing the $\mathrm{pH}$ of the suspension to 5.0 and the ionic strength to 0.05 by adding 1 $\mathrm{mL}$ of concentrated saline acetate buffer to $2 \mathrm{~mL}$ of $1.0 \times 10^{-12} \mathrm{M}$ AuLA (50 nm) suspension in $1 \mathrm{mM} \mathrm{NaOH}$. 


\section{SI-5 Correlation curve of the RLS signal of $50 \mathrm{~nm}$ gold nanospheres measured at $\mathbf{5 2 0} \mathbf{~ n m}$}

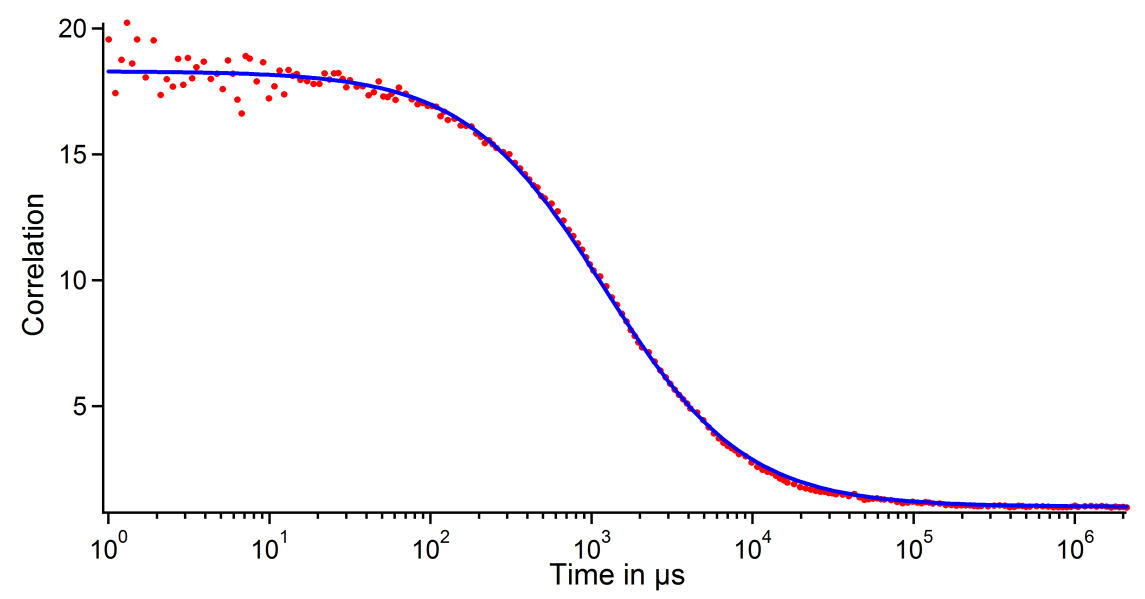

Figure S10. Correlation curve of the light scattering signal from a suspension of 50 $\mathrm{nm}$ gold nanospheres in water, measured at $520 \mathrm{~nm}$. The blue line is a fit to the data using a diffusion time of $1.20 \mathrm{~ms}$. Taking into account the different focal volumes for $520 \mathrm{~nm}$ and $760 \mathrm{~nm}$ light, this diffusion time scales to $2.55 \mathrm{~ms}$ at $760 \mathrm{~nm}$. 


\section{SI-6 Charge-detection mass spectrometry of $80 \mathrm{~nm}$ gold sphere monomers}

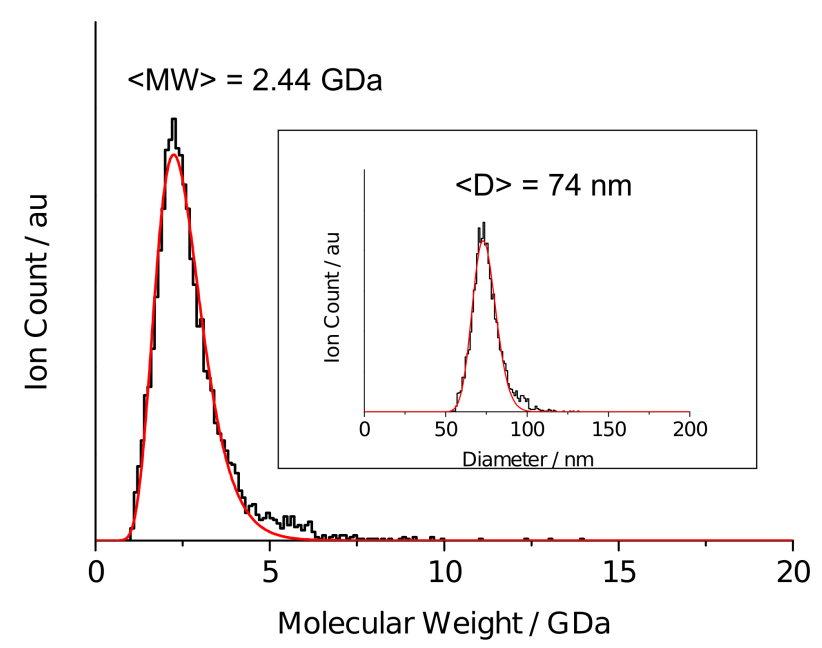

Figure S11. Experimental mass spectrum of $80 \mathrm{~nm}$ diameter gold nanospheres (black line) as measured by CD-MS together with the corresponding log-normal fitting curve (red line). The analytical parameters of the fitting curve give a mean molecular weight of $2.44 \mathrm{GDa}$. In inset is provided the diameter distribution of the sample (black line) assuming a spherical shape for nanoparticles and a density of 19.3 for gold. Similarly the experimental distribution is fitted with a log-normal curve (red line) that gives a mean diameter of $74 \mathrm{~nm}$. 


\section{SI-7 Theoretical calculation of optical cross sections}

\section{SI-7.1 Comparison between DDA and GMM for dimers of gold spheres of $\mathbf{3 0}$ $\mathrm{nm}$}

Optical cross sections calculated for gold nanosphere dimers using DDA (dipole-dipole approximation) theory were compared with data from literature ${ }^{\mathrm{S2}}$ for $30 \mathrm{~nm}$ gold nanosphere dimers calculated using GMM (generalised multiparticle Mie) theory. The published cross section spectra were digitised with the Engauge software package (http://digitizer.sourceforge.net/). The digitalisation reproduces the numerical values of all literature data points with sufficient accuracy.

The data used for the comparisons in Figures S13 and S14 concerns the absorption and scattering cross sections calculated for the excitation polarised along the long axis of the dimer ('longitudinal'). Note that here absorption is considered $\left(\sigma_{\mathrm{abs}}=\sigma_{\mathrm{ext}}-\sigma_{\mathrm{sca}}\right)$, instead of extinction. Also, the values are for a particular orientation (light electric field oriented parallel to long dimer axis), not for random orientation as is the case elsewhere in this work. The cross sections from DDSCAT (which use the 'effective sphere' convention, i.e. $\left.\sigma_{\text {physical }}=2^{2 / 3} \pi a^{2}\right)$ have been converted to the GMM convention $\left(\sigma_{\text {physical }}=2 \pi a^{2}\right.$, see Main Text). The number of discrete dipoles used for the DDA calculations are 763095, 225915 and 226109 for gaps of $0.6 \mathrm{~nm}, 1.5 \mathrm{~nm}$ and $3 \mathrm{~nm}$, respectively. This corresponds to a discrete dipole every $0.34 \mathrm{~nm}, 0.5 \mathrm{~nm}$ and $0.5 \mathrm{~nm}$, respectively. 

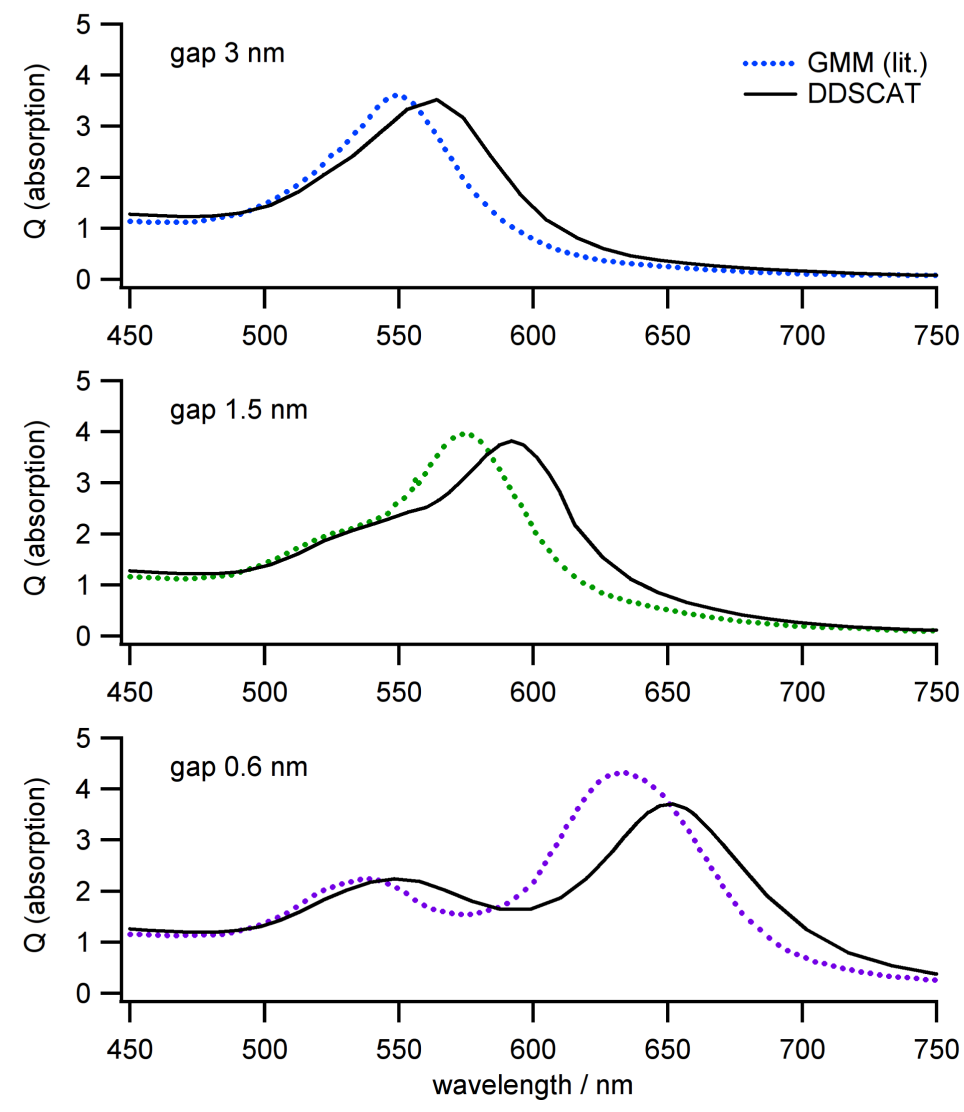

Figure S12. Comparison between the relative absorption cross sections $\left(Q_{\mathrm{abs}}=Q_{\mathrm{ext}}\right.$ $Q_{\text {sca }}$ ) calculated with DDSCAT (solid curves) and GMM (dashed curves) for the longitudinal plasmon resonance of dimers of $30 \mathrm{~nm}$ diameter gold spheres for different interparticle gaps (no orientational average, field polarisation oriented along the long dimer axis).

The absolute values for the absorption cross sections (Figure S13) agree very well between both methods. The peak positions are at slightly shorter wavelengths in the GMM resonances than the DDA resonances. Similar observations hold for the scattering cross sections (Figure S14). For shorter interparticle distance $(0.6 \mathrm{~nm})$, there are differences both in maximum wavelength and in intensity, in particular for scattering. 

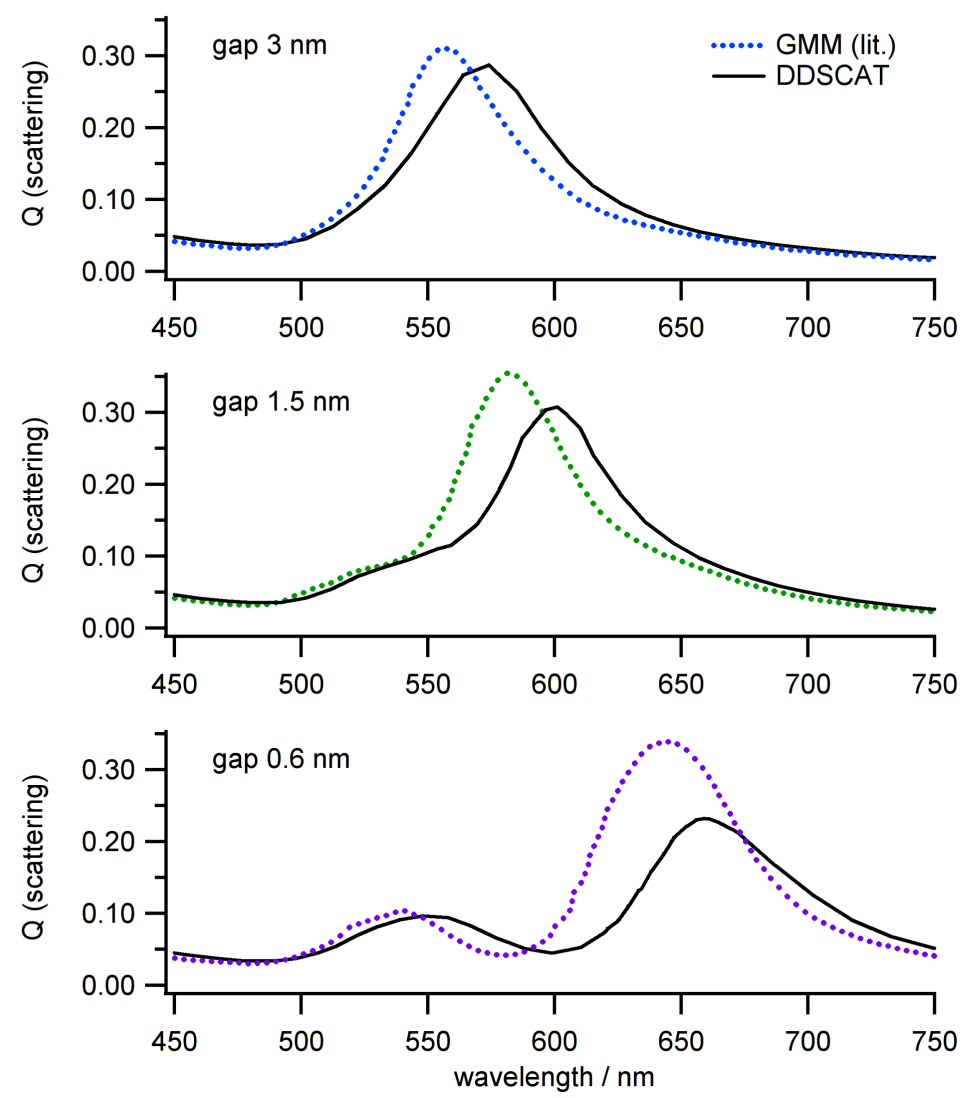

Figure S13. Comparison between the scattering efficiencies calculated with DDSCAT (solid curves) and GMM (dashed curves) for the longitudinal plasmon resonance of dimers of $30 \mathrm{~nm}$ diameter gold spheres for different interparticle gaps (no orientational average, field polarisation oriented along the long dimer axis). 
SI-7.2 Comparison between DDA calculations and experiment for dimers of gold spheres of $20 \mathrm{~nm}, 50 \mathrm{~nm}$ and $80 \mathrm{~nm}$.

SI-7.2.1 Extinction cross section spectra of dimers

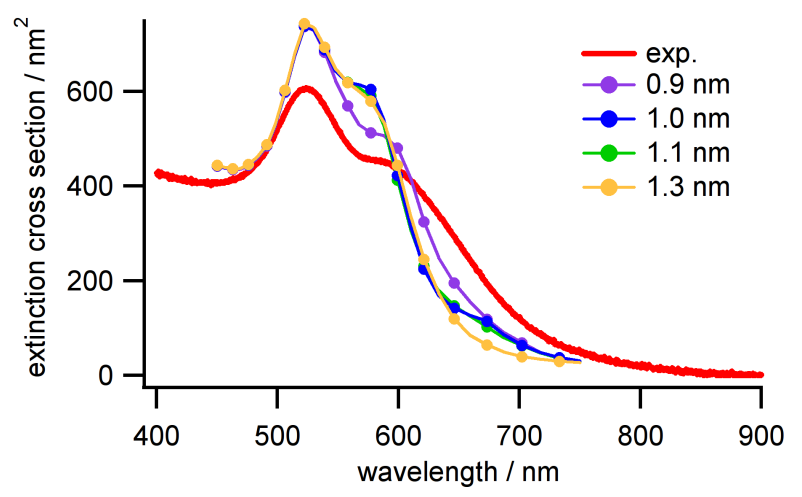

Figure S14. Comparison between the experimental extinction cross sections and the corresponding average DDA extinction cross sections for dimers of $20 \mathrm{~nm}$ gold spheres, for various interparticle gaps, calculated using DDSCAT.

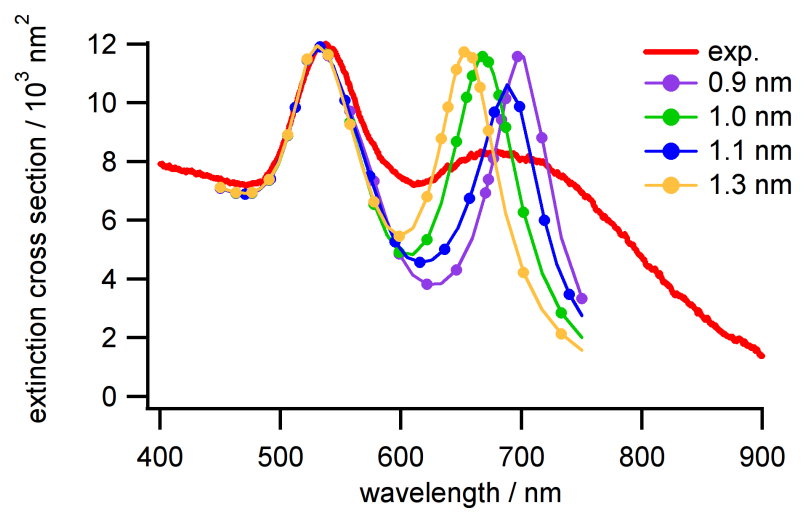

Figure S15. Comparison between the experimental extinction cross sections and the corresponding average DDA extinction cross sections for dimers of $50 \mathrm{~nm}$ gold spheres, for various interparticle gaps, calculated using DDSCAT. 


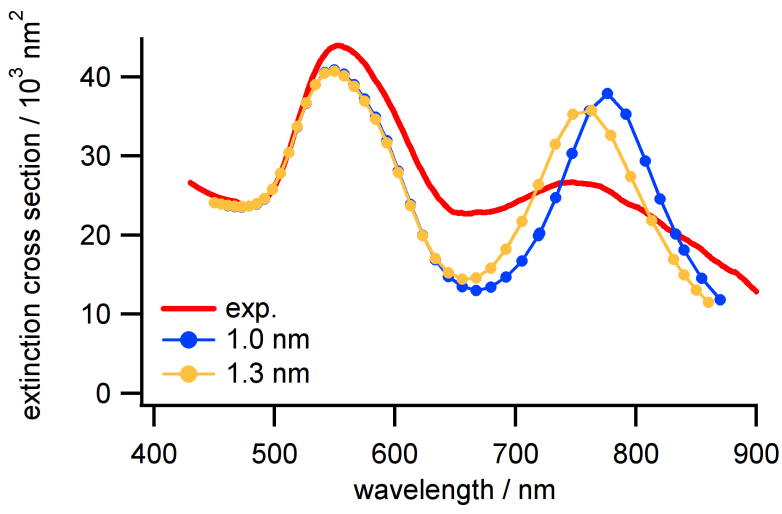

Figure S16. Comparison between the experimental extinction cross sections and the corresponding average DDA extinction cross sections for dimers of $80 \mathrm{~nm}$ gold spheres, for various interparticle gaps, calculated using DDSCAT.

\section{SI-7.2.2 Scattering cross section spectra of dimers}

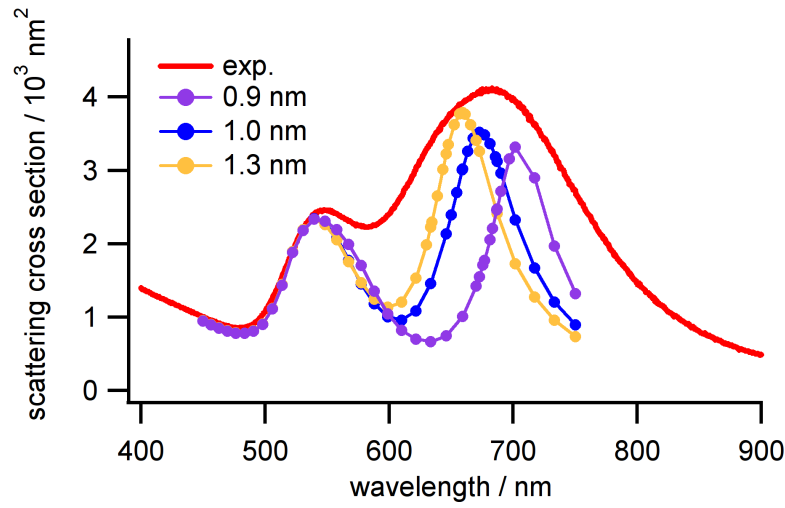

Figure S17. Comparison between the experimental scattering cross sections and the corresponding DDA average scattering cross sections for dimers of $50 \mathrm{~nm}$ gold spheres, for various interparticle gaps, calculated using DDSCAT. 


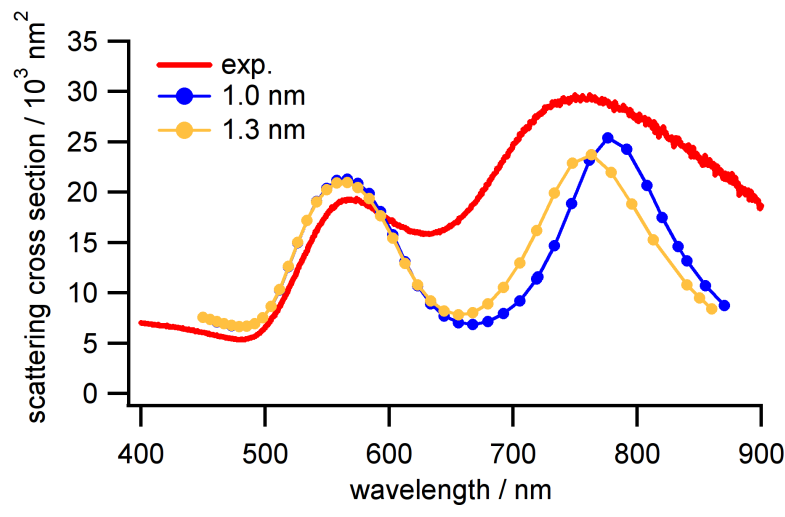

Figure S18. Comparison between the experimental scattering cross sections and the corresponding DDA average scattering cross sections for dimers of $80 \mathrm{~nm}$ gold spheres, for various interparticle gaps, calculated using DDSCAT.

\section{SI-8 References for supporting information}

1. G. Chen, Y. Wang, L. H. Tan, M. Yang, L. S. Tan, Y. Chen, and H. Chen, f. Am. Chem. Soc., 2009, 131, 4218-9.

2. B. Khlebtsov, A. Melnikov, V. Zharov, and N. Khlebtsov, Nanotechnology, 2006, 17, 14371445. 